\title{
Social-aware Opportunistic Routing: The New Trend
}

\author{
Waldir Moreira and Paulo Mendes
}

This is the author's preprint version. Personal use of this material is permitted. However, permission to reprint/republish this material for advertising or promotion or for creating new collective works for resale or for redistribution to thirds must be obtained from the copyright owner. The camera-ready version of this work has been published at Routing in Opportunistic Networks, date of May 2013 and is property of Springer Science+Business Media New York.

Abstract

Since users move around based on social relationships and interests, the resulting movement patterns can represent how nodes are socially connected (i.e., nodes with strong social ties, nodes that meet occasionally by sharing the same working environment). This means that social interactions reflect personal relationships (e.g., family, friends, co-workers, passers-by) that may be translated into statistical contact opportunities within and between social groups over time. Such contact opportunities may be exploited to ensure good data dissemination and retrieval, even in the presence of intermittent connectivity. Thus, in the last years, a new trend based on social similarity emerged where social relationships, interests, popularity and among others, are used to improve opportunistic routing. In this chapter, the reader will learn about the different approaches related to opportunistic routing focusing on the social-aware approaches and how such approaches make use of social information derived from opportunistic contacts to improve data forwarding. Additionally, a brief overview on the existing taxonomies for opportunistic routing as well as an updated one are provided along with a set of experiments in scenarios

Waldir Moreira

SITI, Universidade Lusófona, Campo Grande, 376, Ed. U, 1749-024, Lisboa, Portugal e-mail: waldir.junioreulusofona.pt

Paulo Mendes

SITI, Universidade Lusófona, Campo Grande, 376, Ed. U, 1749-024, Lisboa, Portugal e-mail: paulo.mendesculusofona.pt 
based on synthetic mobility models and human traces in order to show the potential of social-aware solutions.

\section{Introduction}

The increasing capability of portable devices provide users with new forms of communication. They can quickly form networks by sharing resources (i.e., processing, storage) to exchange information and even share connectivity. This is possible through opportunistic contacts among devices carried by these users that can forward information on behalf of other nodes to reach a given destination or connectivity points.

However, opportunistic communication has to cope with link intermittency. Due to such intermittency - which results from node mobility, power-saving schemes, physical obstacles, dark areas (i.e., overcrowded with access points operating in overlapping channels or no infrastructure at all) - no end-to-end path may exist, which consequently causes frequent partitions and high queueing delay.

Solutions based on the knowledge of end-to-end paths perform poorly, and instead, numerous opportunistic routing protocols have been proposed taking advantage of devices capabilities to overcome intermittency. Some opportunistic routing protocols use replicas of the same message to combat the inherent uncertainty of future communication opportunities between nodes 1 . In order to carefully use the available resources and reach short delays, many protocols perform forwarding decisions using locally collected knowledge about node behavior to predict which nodes are likely to deliver a content or bring it closer to the destination (cf. Fig. 11). For that, nodes must have enough processing power and storage to keep data until another good intermediate carrier node or the destination is found [5], following a store-carry-and-forward (SCF) paradigm.

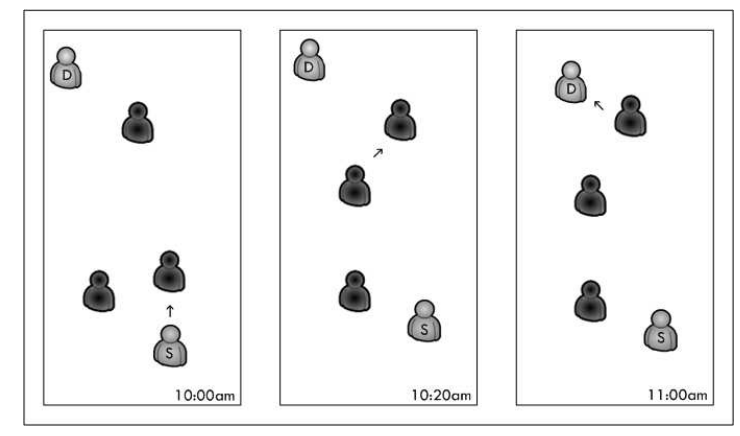

Fig. 1 Example of opportunistic routing

\footnotetext{
${ }^{1}$ For the sake of simplicity, node and user are used interchangeably throughout this chapter.
} 
Fig. 1 depicts how content opportunistically reaches its destination by being transferred and temporarily stored among nodes.

Proposed approaches range from using node mobility to flood the network for fast delivery (e.g., Epidemic [39]) up to controlling such flooding to achieve the same results based on: encounter history (e.g., PROPHET [23, 11]), optimized delivery probability (e.g., Spray and Wait [35]), prioritization (e.g., MaxProp [4]), and encounter prediction (e.g., $E B R[30]$ ).

Since 2007, a trend has emerged based on different representations of social similarity: i) labeling users according to their work affiliation (e.g., Label [15]); ii) looking at the importance (i.e., popularity) of nodes (e.g., PeopleRank [29]); iii) combining the notion of community and centrality (e.g., SimBet [8] and Bubble Rap [17]); iv) considering interests that users have in common (e.g., SocialCast [7]); v) inferring different level of social interactions (e.g., dLife [28]) or predicting future social interactions (e.g., CiPRO [31]) from the users' dynamic behavior found in their daily life routines.

These social-aware opportunistic routing solutions have shown great potential in what concerns information delivery since i) cooperation among users sharing social aspects (e.g., relationship, tastes, interests, affiliation, ...) is encouraged, which is beneficial to improve content dissemination [18], and ii) social information is much less volatile than human mobility, providing more robust and reliable connectivity graphs that aid routing [14, 17].

Thus, with this chapter the reader is expected to learn about the different opportunistic routing solutions with emphasis on the social-aware approaches and how such approaches use social information to improve data forwarding. Additionally, this chapter provides a brief overview of existing opportunistic routing taxonomies, so the reader understands how social similarity has gained attention in the last years. And, a set of experiments is presented to show that proposals based on social similarity indeed have a good potential to improve forwarding in opportunistic networks.

This chapter is structured as follows. Section 2 presents the relevant previous work that are oblivious to social information. Then, the social-aware opportunistic routing approaches are identified in Section 3 followed by a brief discussion on the existing taxonomies for opportunistic routing in Section 4 which makes a reference to when social similarity started to be considered for routing improvements. Next, Section 5 presents some results to show the gains of the social-aware approaches over the social-oblivious ones, and Section 6 concludes the chapter.

\section{Social-oblivious Opportunistic Routing Approaches}

According to Jain et al. [19], deterministic routing approaches are excellent from a performance point of view, since the more information a node can get from the network, the wiser its forwarding decision will be. However, the needed extra knowledge brings more complexity to the solution and even makes it impossible to be implemented due to the dynamic nature of user behavior. It was already shown that, 
although the optimal solution will need to have a broad knowledge about the network behavior and traffic demands [19], even the most simple oracle, called Contact Oracle, which contains information about contacts between any two nodes, is unrealistic as it is equivalent to knowing the time-varying characteristics of such networks. Other oracles were defined, including a Queuing Oracle (i.e., information about instantaneous buffer occupancies), and a Traffic Demand Oracle (i.e., information about the present or future traffic demand), but their assumptions about the network behavior is even more severe. The existence of such oracles would support deterministic routing algorithms able to compute an end-to-end path (possibly time dependent) before messages were actually transmitted. For example, if a Contact Oracle is available, modified Dijkstra with time-varying cost function based on waiting time would be enough to find the route. However, the most realistic assumption is that network topology is not known ahead of time.

Based on the analysis of deterministic routing approaches, it is clear that the most suitable solution would be the one based on a local probabilistic decision, aiming to forward messages based on the opportunities raised by any contact within range. More elaborated solutions may also take other information into account to increase the efficiency of message progression towards a destination. Examples of such extra information are: history data about encounters, mobility patterns, priority of information, and social ties.

In order to understand the importance of social similarity for opportunistic routing and its applicability, this section provides an overview of the most relevant proposals to perform opportunistic routing spanning a 11-year period (2000-2011) and aims at contributing to a broad understanding of existing opportunistic routing approaches prior to the social-aware solutions. These routing proposals are grouped into three categories: single-copy, aiming to improve the usage of network resources; epidemic, aiming to increase delivery probability; and, probabilistic-based, aiming to find an optimal balancing between both previous categories.

\subsection{Single-copy Routing}

In resource-constrained networks, which can occur in urban areas with high spectrum interference, opportunistic routing may lead to waste of resources when trying to deliver messages to a destination or set of destinations. Such waste of resources is mainly due to the utilization of message replication aiming to increase delivery probability.

Aiming to optimize the usage of network resources, some approaches avoid replication of messages, forwarding messages to single next-hops based on available connectivity and some form of mobility prediction. This means that these proposals perform single-copy forwarding, i.e., only one copy of each message traverses the network towards the final destination. Such copy can be forwarded if the node carrying it decides (i.e., randomly, or based on a utility function) that another encountered node presents a higher probability to deliver the message. 
Minimum Estimated Expected Delay (MEED) [20] is an example of a single-copy forwarding approach that uses contact history (i.e., connection and disconnection times of contacts) to aid forwarding. Contact history is a metric that estimates the time a message will wait until it is forwarded. A per-contact routing scheme is used to "override" regular link-state routing decision. That is, instead of waiting for nodes enclosed in the path with the shortest cost (based on MEED values), it simply uses any other contact opportunity (i.e., node) that arises prior to what is expected to forward the message. To be able to do this, MEED must recompute routing tables each time a contact arrives and also broadcast this information to every other node in the network.

Spyropoulos et al. (2008) [37 present six examples where this type of forwarding is considered: i) direct transmission, where messages are forwarded only to the destination; ii) randomized routing, where messages are only forwarded to encountered nodes that have forwarding probability $p$, where $0<p \leqslant 1$; iii) utility-based routing with 1-hop diffusion, where forwarding takes place if the utility function based on encounter timers - of an encountered node is higher than the one of the current carrier; iv) utility-based routing with transitivity, in which an utility function towards a destination is updated also considering intermediate nodes that have high utility to such destination; v) seek and focus routing, where randomized routing is used to find the best starting point towards the destination and henceforth a utility-based approach is used to find the destination; and vi) oracle-based optimal algorithm, with which the future movement is known beforehand allowing optimal forwarding decisions to be taken aiming to delivery messages in a short amount of time.

From a resource consumption viewpoint, single-copy forwarding approaches are quite interesting, since they keep the usage of network (e.g., bandwidth) and node (e.g., energy, storage) resources at a low level. However, they suffer from high delay rates which, consequently, may result in a low delivery ratio. Another issue is related to the amount of knowledge that needs to be exchanged/available in order to aid forwarding, which in some scenarios generates too much overhead and may be impossible to implement.

To mitigate such problem, the following category of opportunistic routing approaches relies only on current contacts to increase delivery probability.

\subsection{Epidemic Routing}

Ubiquitous communication is a feature that has been present in our everyday life and requires messages to be delivered with high probability to their destinations, with the help of intermediary nodes able to implement the SCF paradigm.

Since networks are created by people moving around, opportunistic contacts are considered to increase delivery probability in networks with the aforementioned characteristics in a proposal called Epidemic [39]. With the Epidemic routing approach, every node in the network gets at least a copy of each message. Such a full 
replication strategy leads to an increase of the delivery rate. Replication of messages is done by means of summary vectors that are exchanged between nodes upon a contact. Such summary vectors contain the list of messages each node is carrying, allowing nodes to exchange all messages that the other node is lacking. The proposal indeed increases delivery rate, since every potential forwarder has, with high probability, a copy of the message, assuming contacts with significant duration and sufficient buffer space in each node. However, to avoid waste of resources, each host sets a maximum buffer size that it is willing to allocate for epidemic message distribution. In general, nodes drop older messages in favor of newer ones upon reaching their buffer's capacity. This means that the efficiency of the delivery process depends upon the configured buffer space, which may be substantially improved with the usage of Bloom filters [3]. In order to avoid messages to be replicated indefinitely, a hop count field determines the maximum number of epidemic exchanges that a particular message is subject to, being messages dropped based on the locally available buffer space. Since the number of hops towards the destination is not known in advance setting a hop count may decrease the delivery probability. A stale-data removal mechanism could be more efficient by removing messages that were already delivered.

In an attempt to avoid waste of network resources, other proposals emerged based on a controlled replication approach. That is, the number of nodes which get a message copy is reduced by probabilistically choosing next nodes or by using a utility function.

\subsection{Probabilistic-based Routing}

Probabilistic approaches are based on the estimation/prediction of what is the next best set of carriers for each message based on some probability metric aiming to maximize delivery probability. Probabilistic forwarding protocols require node mobility patterns that exhibits long-term regularities such that some nodes consistently meet more frequently than others over time: the mean inter-meeting time between two nodes in the past will be close to that in the future with high probability.

Within this category, proposals attempt, first of all, to optimize the delivery probability while avoiding full replication of messages. Besides this core concern, there are proposals that take into account the capabilities of nodes, the priority of messages and the availability of resources aiming to achieve a high delivery ratio with low delay and resource consumption. Another concern of some probabilistic-based approaches is to use as few meta-data as possible aiming to decrease concerns with respect to energy, processing and bandwidth saving. Having considerable control overhead increases contention in the network resulting in message discarding and retransmissions.

Since 2003, different delivery probability metrics have been proposed including frequency encounters [23, 11, 4, 34, 30], aging encounters [10, 12, 36], aging messages [35, 24], and resource allocation [32, 1]. 


\subsubsection{Frequency Encounters}

Proposals based on these metrics have in common the fact that they rely on the knowledge about how many times nodes meet in a network. The proposals considered in this sub-category are the Probabilistic ROuting Protocol using History of Encounters and Transitivity (PROPHET), MaxProp, Prediction, and EncounterBased Routing (EBR).

One of the most well known approaches, being currently considered by the DTN Research Group (DTNRG) of the Internet Research Task Force (IRTF), is the Probabilistic ROuting Protocol using History of Encounters and Transitivity (PROPHET) [23, 11]. PROPHET uses a probabilistic metric called delivery predictability, which indicates what is the likelihood of a node to deliver a message to a destination based on its past contacts with such destination. If a pair of nodes does not encounter each other for a while, they are less likely to be good forwarders of messages to each other, thus their delivery predictability is reduced in the process. Transitivity is a property of this predictability where if a node $A$ regularly meets a node $B$ and node $B$ regularly meets node $C$, this implies that node $C$ is also a good node to forward messages to node $A$. Delivery predictability helps to decide whether the node should replicate or not a given message (It is worth mentioning that delivery predictability was changed to cope with the "parking lot" problem as reported by Grasic et al. (2011) [11]). Upon a contact, nodes exchange summary vectors that also have information regarding delivery predictability. This information is used to update their own delivery predictability vectors, which are used to make decisions about message forwarding. PROPHET delivers messages only to nodes which are better (in terms of delivery predictability) than the current carrier node, resulting in a reduction of the consumption of network resources and a high probability of messages delivery.

However, flooding can still occur with PROPHET if the message is originated in a node with low delivery predictability (i.e., node with low mobility) towards the destination, and this node only encounters nodes with higher delivery predictability.

MaxProp [4] is another probabilistic-based approach that uses a metric called delivery likelihood of messages, by having each node keeping track of a probability of meeting any other peer. Using an incremental averaging method, nodes that are seen frequently obtain higher delivery likelihood values over time. Each time two nodes meet, they exchange their delivery likelihood probabilities towards other nodes. Based on the delivery likelihood values computed by other nodes and by itself, a carrier of a message computes a cost for each possible path to the destination, up to $n$ hops. The cost for a path is the sum of the probability of each contact on the path not occurring. This cost estimation, along with the hop count, are then used to order messages for scheduling and for dropping. In addition, MaxProp assigns a higher priority to new messages (i.e., low hop count) to increase their chance of reaching the destination faster, and tries to prevent reception of the same message twice by including a hop list in each message, and uses acknowledgments to notify all nodes about message delivery. Upon contact, two nodes exchange messages in a specific priority order: first, messages that have these nodes as final destinations; 
second, information for estimating delivery likelihood; third, acknowledgments to remove stale messages; fourth, messages that have not traversed far in the network; and, fifth, send messages with highest priority.

By combining the estimation of message delivery likelihood with message priority and acknowledgments, MaxProp is able to reach good performance regarding message delivery probability and message latency with transfer opportunities limited in duration and bandwidth. However, it is considered that nodes have unlimited storage for their own messages and limited storage for the messages coming from other nodes, which has an impact on the overall performance.

It is important to mention that the delivery likelihood metric used in MaxProp is different from the delivery predictability metric employed in PROPHET in the sense that the former depends solely on the probability of nodes to meet each other while PROPHET depends on the probability to meet the destination itself, which means that PROPHET requires more state. However, MaxProp requires nodes to compute possible paths to the destination by concatenation of delivery probability between nodes while PROPHET does not require further computation, since messages are forwarded if a node has higher delivery predictability towards the destination than the carrier.

Song and Kotz proposed a prediction-based approach (hereafter referred to as Prediction) [34] that makes use of contact information to estimate the probability of meeting other nodes in the future. As happens with PROPHET and MaxProp, Prediction also uses historical contact information to estimate the probability of meeting other nodes in the future. However, unlikely previous approaches, Prediction estimates the contact probability within a period of time, based on a metric called timely-contact probability, which is used to compute the contact frequency between two nodes, as follows: the contact history between two nodes $i$ and $j$ is divided into a sequence of $n$ periods of $\Delta T$ starting from the start time $\left(t_{0}\right)$ of the first contact in history to the current time. If node $i$ had any contact with node $j$ during a given period $m$, which is $\left[t_{0}+m \Delta T, t_{0}+(m+1) \Delta T\right]$, the contact status of the interval $I_{m}$ is set to 1 . The probability of node $i$ meeting node $j$ in the next $\Delta T$ can be estimated as the average of the contact status in prior intervals. In Prediction, whenever two nodes meet, they exchange the indexes of all their messages. If the destination of a message is not the node in contact, the probability to deliver such message through that node is computed. If the probability of delivering the message via the contacted node (based on the past average number of encounters with the destination) within a defined period of time is greater than or equal to a certain threshold, the message is passed to the node in contact. This proposal presents two methods for choosing the next node: the decision can be taken by the node that is sending the message or by the one which is receiving it. In the former case, a meta-data message is necessary to determine if the receiver is a good next hop. As for the latter, the receiver decides whether or not to keep the copy of the message considering its own probability of coming into contact with the destination.

Prediction achieves good performance regarding message delivery with low number of message transmission and duplication. However, its performance and storage usage is directly proportional to the Time-To-Live (TTL) allowed for mes- 
sages which makes it more suitable for networks tolerant to very long delays (i.e., sparse networks).

The proposal Encounter-Based Routing (EBR) [30] also considers the number of times nodes meet in order to predict the rate levels of future encounters. It simply counts the number of contacts a node has with other nodes (Current Window Counter) and determines the Encounter Value (EV) that represents the node's past rate of encounters. The higher EV is, the higher the probability of successful message delivery. This also determines the number of replicas of a message that the relay node will get in each contact. Nodes maintain their past rate of encounters to predict their rate of future encounters. When nodes meet, they first update their $\mathrm{EV}$ values and estimate the EVs ratio, which is used to determine the number of tokens of a message replica that will be passed to each neighbor. This is a kind of time-to-live parameter also used by other approaches such as Spray and Focus. For security reasons, prior to EV update, nodes exchange information that will aid them to determine if their EV values are correct.

The prediction of future encounters used by $E B R$ allows the improvement of latency and message delivery by reducing traffic overhead (i.e., unwanted copies). Messages are only exchanged with nodes that have high encounter rate, which avoids routes that may not result in delivery, and minimizes network resource usage. In addition, the proposal implements a security measure that avoids black hole denial-of-service attacks from malicious nodes pretending to be part of the network and announcing fake EV values. However, this approach presents some drawbacks in scenarios with multiple communities that have low rate of inter-contact times. In these scenarios, messages may be forwarded to nodes that indeed have higher EV values, but such messages will get stuck within the source community. Also, this security measure incurs in wasting contact opportunities for determining the reliability of the encountered node, and the proposal may have its performance degraded in scenarios where nodes have short contact times.

\subsubsection{Aging Encounters}

In this sub-category, the age of encounters are taken into consideration, and the proposals that fall into it are the Exponential Age SEarch (EASE), FResher Encounter SearcH (FRESH), and Spray and Focus.

The Exponential Age SEarch (EASE) proposal [12], first presented in 2003, was one of the first proposals to consider history of encounters with a specific destination to support opportunistic forwarding. In addition to that, EASE also considers geographic position of nodes where a node would make routing decisions based on the time and location of its last encounter with every other node in the network. With EASE, every node maintains local information about the time and location of its last encounter with other nodes in the network. To be considered a good next hop, a node must either have a more recent encounter with the destination than the current holder of the message or the node must be physically closer to the destination. Once the new hop is found, any position-based algorithm (e.g., DREAM [2]) can 
be employed to route the message towards it. These two phases, namely next hop search and message routing, are repeated until the best next hop is the destination itself. A second version of the proposal (Greedy EASE [12]) is able to change the chosen next hop during the routing phase as it checks the age of the last encounter with the destination at every hop.

EASE performs quite well in scenarios with nodes presenting random walk mobility patterns. The performance results show that the routes towards the destination have the same length (sometimes smaller) as the optimal case even for very large distances between the communicating nodes. However, this proposal is highly dependent on the mobility pattern and destination speed. Its performance is easily degraded if the destination moves fast turning the solution more costly (i.e., in terms of route length). Added to that, if mobile nodes shutdown their wireless interface (for energy-saving purposes), estimates considering location are of no use.

The FResher Encounter SearcH (FRESH) [10] proposal is an example of a "blind" routing protocol, since it has no notion of coordinates. Each node keeps track of the time elapsed since the last encounter with every other node, and uses this information to choose the next hop for message forwarding. When a sender wishes to initiate data forwarding, it must first search for the next hop that is determined by the time elapsed since the last encounter between this potential next hop and the destination. This search is omni-directional and is done in concentric rings with increasing radius until the next hop is found. It is necessary that nodes keep track of their one-hop neighbors to maintain encounter tables updated.

FRESH takes advantage of the time-distance correlation where the distance traveled during a time interval of duration $t$ is positively correlated with $t$ (e.g., a node met a few minutes ago is closer than a node met two hours before). With that, it is able to improve the performance of route discovery with no need for global knowledge of the network. Instead, the proposal is based on a distributed implementation where next hop search is defined in terms of local information (e.g., encounter tables). Like with $E A S E$, performance depends on nodes mobility processes as the time-distance correlation becomes noisier with heterogeneous speeds. That means that a node that has just encountered the destination may not be close to it if the destination is moving too fast. Another issue is that FRESH may suffer with loops in routing if source and destination are not part of a connected subset of nodes. This is indeed a problem especially in scenarios where isolated cluster of nodes may be formed. There is also an overhead related to the need of having one-hop neighbor encounter table updated.

The more recent Spray and Focus [36] approach proposes a scheme were a fixed number of copies are spread initially exactly as in Spray and Wait [35] (with the subtle difference of using only $\frac{1}{3}$ or $\frac{1}{2}$ of the $L$ messages normally used in Spray and Wait), but then each copy is routed independently according to single-copy utility-based scheme with transitivity [37]. In the spraying phase, ideally it would be good to be able to choose as relays the $L$ nodes that most frequently encounter the destination. However, waiting for a "better" relay may mean that opportunities to spread extra copies are forfeited. Hence, the Spray and Focus scheme uses a greedy spraying phase by implementing a binary spraying algorithm to minimize 
the amount of time it takes to spray all $L$ copies, moving the problem of looking for a possibly better relay to the focus phase. In the focus phase, each potential router maintains a timer for every other node in the network, recording the time elapsed since the two nodes came within transmission range for the last time. These timers are similar to the age of last encounter [10] and contain indirect location information. For a large number of mobility models, it can be shown that a smaller timer value on average implies a smaller distance from the node in question.

Spray and Focus outperforms flooding-based (i.e., Epidemic) and single-copy schemes (i.e., Randomized Flooding, and Utility-based Flooding) as well as the other spraying algorithm (i.e., Spray and Wait) under realistic mobility scenarios (e.g., modeling human behavior), by forwarding messages to nodes which have a "closer" relationship (determined by the encounter timers) with the destination. Also, Spray and Focus presents good performance in scenarios with heterogeneous mobility using an algorithm that is able to diffuse timer information much faster than regular last encounter based schemes. However, since its performance is highly dependent on the use of encounter timers, it can be easily degraded in scenarios where nodes are highly mobile as timers quickly become obsolete.

\subsubsection{Aging Messages}

These proposals have in common the fact that they aim to avoid messages to be kept being forwarded in the network by creating metrics that define the age of message copies. Spray and Wait, and Optimal Probabilistic Forwarding (OPF) are the proposals comprised by this sub-category.

One of the first proposals was Spray and Wait [35], which decouples the number of transmissions per message from the total number of nodes, generating a small number of transmissions in a large range of scenarios. Initially, copies of a message are spread quickly in a manner similar to epidemic routing. However, Spray and Wait stops when enough copies have been sprayed in order to avoid flooding, while guaranteeing that at least one copy will reach the destination with high probability. By exploiting the mobility of nodes, Spray and Wait operates in two steps: first, the source determines a certain number of adjacent nodes that are going to get a copy of the message (spraying phase). In the second step, the nodes that got a copy of the message deliver it directly to the destination when it gets within range (waiting phase). Each generated message can have $L$ copies of it distributed in the network. This number of copies can be determined in two ways: i) based on the number of nodes $M$ and size of the network $N$; ii) by estimating $M$ when both $M$ and $N$ are unknown. Once $L$ is known, the source can spread the copies of the message by passing only one copy of the message (Source Spray and Wait), or $L / 2$ copies (Binary Spray and Wait) to each encountered node. In the latter case, the receiver of $L / 2$ copies will spread the obtained copies in the same way. If the destination is not found in the spraying phase, the nodes holding one copy of the message will forward it directly to the final destination. 
Spray and Wait exploits node mobility being able to limit the total number of copies and transmissions per message resulting in an energy-efficient solution with low delivery delay, although the achieved delay is inversely proportional to the number of copies. If nodes move quickly enough around the network, Spray and Wait shows that only a small number of copies can create enough diversity to achieve close-to-optimal delays. However, there is no acknowledgment mechanism to get rid of copies of already delivered messages and no mechanism to select the best set of forwarders in the spraying phase. In what concerns computational effort, determining $L$ is not an easy task, since it is necessary to know $M$ beforehand and it depends on nodes performing independent random walks. This can easily result in an inaccurate measure of $L$, which degrades the algorithm's performance. This problem is even worse in large dense networks with frequent disconnections and nodes following different mobility patterns.

The Optimal Probabilistic Forwarding (OPF) [24] protocol replicates a message upon node encounter if, by doing so, such action increases the overall delivery probability of such message. That is, if this action maximizes the joint expected delivery probability of the copies to be placed in system (i.e., in the sender and receiver nodes of the message). $O P F$ aims to maximize the delivery probability based on a particular knowledge about the network, relying on the assumptions that node mobility exhibits long-term regularity (enabling the estimation of mean inter-meeting times) and that each node knows the mean inter-meeting time of all pairs of nodes in the network. $O P F$ metric reflects not only the direct delivery probability of a message, such as in PROPHET, but also the indirect delivery probability when the node can forward the message to other intermediate nodes, as in MaxProp. However, unlike MaxProp, OPF metric reflects a hop-count-limited forwarding scheme, based on a function of two important states of a message: remaining hop-count and residual lifetime. Such utility function may estimate the effect that message replication may have on the expected delivery rate while satisfying the constant on the number of forwardings per message ( $O P F$ has a performance awareness as happens with $R A P I D$, for instance).

With $O P F$ every message has a residual time-to-live $\left(T_{r}\right)$ that also denotes a given meeting time slot, and nodes know the mean inter-meeting time $\left(I_{i, j}\right)$ between any two nodes $i$ and $j$ in the network. This is used to determine the meeting probability $\left(M_{i, j}\right)$ among any two nodes and the delivery probability $\left(P_{i, j, K, T_{r}}\right)$ between nodes $i$ and $j$ of a message with $K$ remaining hop-count and $T_{r}$ residual time to live. The delivery probability is simply given by $P_{i, d, 0, T_{r}}$ if the message cannot use anymore hops to reach the destination. However, when the message is at $K$ hops from the destination, forwarding will take place if the combined probability of the two new copies of the message at the next time-slot $T_{r-1}$ (i.e., $1-\left(1-P_{i, d, K-1, T_{r-1}}\right) \times(1-$ $\left.P_{j, d, K-1, T_{r-1}}\right)$ ) is greater or equals to the probability of not forwarding it at all (i.e., $\left.P_{i, d, K, T_{r-1}}\right)$. So, when node $i$ meets node $j$, whether $i$ should forward the copy to $j$ depends on whether replacing the copy in $i$ with two logically new copies (i.e., in $i$ and $j$ ) increases the overall delivery probability. $O P F$ also comes in another version where $K$ is then substituted by the number of logical tickets ( $L$, as in Spray 
and Wait) which are going to be distributed between the two replacing copies in a message forwarding.

$O P F$ is able to achieve good overall delivery rate with a subtle increase in delay since it only forwards messages to really good relay nodes. Its performance is better if the relationship between nodes is greater and the hop count $(K)$ allowed for each message is chosen wisely. However, this good performance comes with a cost, since it is really dependent on the amount of routing information available. In networks where only local information is available due to the dynamicity of nodes, $O P F$ will have its performance degraded since it needs the mean inter-meeting time of all nodes in the network and such information may be difficult to obtain. Added to that, since the mobility model considered follows an exponential inter-meeting time, the measurements may not represent human behavior as it is known that a power law distribution better represents such behavior [14].

\subsubsection{Resource Allocation}

In what concerns approaches that are aware of available resources, we have the Resource Allocation Protocol for Intentional DTN (RAPID), and PRioritized EPidemic (PREP).

The proposal dubbed Resource Allocation Protocol for Intentional DTN (RAPID) [1] opportunistically replicates messages based upon a utility function that estimates the effect that message replication may have on a predefined performance metric in a network with resource constraints. When nodes meet, they exchange meta-data about messages to be delivered and acknowledgements about already delivered messages, along with messages destined to each other. With the meta-data, nodes are able to determine the marginal utility (which must have the highest increase based on the performance metric considered) of replicating messages between them. RAPID calculates the effect of replication considering resources constraints by exchanging meta-data through an in-band control channel that allows it to have a global state of the network resources (e.g., length of past transfers, expected meeting times, list of delivered messages, delivery delay estimate for buffered messages, changed information on messages since last exchange).

$R A P I D$ has a small cost in the usage of contact opportunities due to the utilization of an in-band control channel. Moreover, the information exchanged in such channel may not always be updated due to node mobility, delivery delay, and unacknowledged messages. This cost may be very high in bandwidth-constrained scenarios with short-lived contact opportunities. Besides that, there are no overall performance guarantees since heuristics are based on sub-optimal solutions supported by one metric at a time. In addition, the performance is also related to the used mobility pattern (e.g., predictable vehicular movements) and can be degraded in scenarios with unpredictable mobility patterns.

In what concerns metric-based approaches, the PRioritized EPidemic (PREP) [32] proposal is also based on message prioritization and the idea of prediction. PREP introduces the average availability (AA) metric that measures the average 
fraction of time a link will be available in the future (i.e., the inter-node cost) and defines drop and transmit priorities (in which lower values indicate high priority) for each message. By using a regular discovery algorithm, each node finds out about its links towards neighboring nodes. According to available information about the past state of the link (i.e., up/down), a node can determine the availability of the links for future use. Then, costs are assigned to links based on their AA values and epidemically broadcasted in the network. To find the lowest cost path to a destination (or its whereabouts), the Dijkstra's shortest path algorithm is employed. Any changes to AA values will trigger link costs updates, which are again broadcasted through Link State Advertisements.

PREP is able to generate a gradient of message replication density that is inversely proportional to the distance towards the destination. That is, messages' copies are kept as close as possible to their destinations. PREP sets a drop priority where messages shall be discarded, upon a full buffer, according to a cost determined by the distance between the message's holder and destination. The greater the distance, the higher the drop priority. In addition, a transmission priority is also set to messages considering their expiry time and the cost previously mentioned. This allows a wiser usage of resources (e.g., storage and bandwidth) having a good effect on message delivery. However, in dynamic scenarios, PREP has its performance degraded since its delivery capability is inversely proportional to the level of disruption happening in the scenario.

\subsubsection{Considerations}

This section introduced the reader with few social-oblivious opportunistic in order to show their advantages and disadvantages. With this, we expect the reader to understand the need to incorporate social information in opportunistic routing has emerged as nodes area carried by human who happen to have social similarities (e.g., same workplace, shared interests, ...). It is important to note that the presented solutions were chosen according to the: i) number of times they have been referenced (i.e., served as benchmarks); or ii) number of benchmarks they have used for their evaluation.

We must highlight that some authors may consider some of the aforementioned solutions as social-aware (for considering the history of encounters, for instance). However, it is our belief that social-aware solutions are those which have much more elaborate utility functions and/or consider features that can be used to identify/classify individuals or groups of these, i.e., common affiliations, shared interests, social ties, popularity, centrality, among others which will be further discussed in Section 3 


\section{Social-aware Opportunistic Routing}

Within the previous solutions for opportunistic routing, one of the processes that may lead to significant consumption of energy, processing capability and bandwidth is the prediction of mobility patterns, which is quite common to all probabilistic routing approaches. One alternative may be to devise probabilistic solutions that exploit not only mobility of nodes but also their social similarities. The reason is that mobility patterns change faster (causing the appearance of unwanted traffic due to out-of-date information) than social relationships between people within a society. The fact that social relationships are less volatile than mobility behavior has been proven [14, 17] to be rather useful in forwarding decisions. Additionally, the higher social similarity, the better content dissemination is [18]. Hence, since 2007 several approaches have been investigating the exploitation of social aspects such as relationships, interests, common affiliations, in order to improve the delivery rate while decreasing the consumption of network resources. The proposals that are part of this sub-category are Label, SimBet, Bubble Rap, SocialCast, PeopleRank, dLife, and $C i P R O$.

The Label [15] approach was one of the first proposals to employ social characteristics into opportunistic routing. Experiments were conducted in INFOCOM 2006 where nodes were labeled telling others about their affiliation/group, and this allowed nodes to forward messages directly to destinations, or to next hops belonging to the same group (i.e., same label) as the destinations. The proposal looked not only to inter-contact time distribution for all the nodes inside a group but also to the inter-contact time distribution between two groups (i.e., friendship ties) with results showing that nodes from one group may be good forwarders for nodes in the corresponding friendship group. The Label proposal provided the first indication that exploiting social similarities improves delivery ratio and especially delivery cost (i.e., total number of messages and duplicates transmitted). Another observation is that friendship between different communities (i.e., unusual connections among nodes of both communities) can be used to slightly improve delivery ratio.

Label's performance is directly related to the allowed message TTL and the mixing rate of nodes in the scenario. Delivery ratio is very low in the case of messages with short TTL, and it is easily degraded if nodes do not mix well. The reason is that Label performs only one-hop delivery and just to nodes belonging to the same community as the destination, which means that delivery may fail if the sender never encounters members of the same community as the destination.

Some network nodes may have such a behavior that make the usage of encounters inefficient to forward messages, due to their sporadic meeting rates. For instance, a node may be involved in a highly clustered network in which none of the nodes have directly or indirectly met the destination node. However, paths between clusters may be insured by nodes that form bridges based on weak acquaintance ties. In this context, the SimBet [8] approach proposes to forward data based on the identification of these bridges and the identification of nodes that reside within the same cluster as the destination node. The major contribution is a new forwarding metric based on ego network analysis to locally determine betweenness centrality of nodes (i.e., 
importance of a node in the system, defined as the number of connections between nodes belonging to different communities that cross the referred node) and social similarity (i.e., probability of future collaboration between nodes in the same community). These two social parameters are determined from an adjacency matrix that each node keeps to track contacts (direct and through neighbor nodes) with other nodes in the network. When a node $i$ meets a node $j$, they exchange messages they have to each other and request each other's list of contacts. With this list, they can update their own contact list along with their betweenness (Bet) and similarity (Sim) values. Then, they exchange summary vectors that contain the destinations to which they are carrying messages along with their updated Bet and Sim values. For each destination in the summary vector, they determine their SimBet utility. With this, a vector of destinations is created containing all the destinations to which the node has highest SimBet utility. This vector of destinations is exchanged and nodes exchange messages to destinations present in their own vector and remove such messages from their buffers.

SimBet is able to forward messages even if the destination node is unknown to the sending node or its contacts. In this case, the message is routed to a structurally more central node where the potential of finding a suitable carrier is much higher. Moreover, SimBet makes no assumptions about the control of node movements or knowledge of node future movements. Finally, with SimBet messages are forwarded solely based on locally obtained information. It works based on forwarding a single copy of each message in the network, which makes it able to reduce resource consumption, mainly buffer space and energy. It has good overall performance regarding message delivery, which is close to Epidemic's but with highly improved delivery cost (i.e., very low number of required forwards to reach destination). However, this proposal may suffer with high delay since the level of contact (i.e., how often and with whom nodes meet) between nodes is a key aspect regarding dissemination of information. That is, if this level of contact between nodes is low, information (e.g., Sim and Bet values, contact lists) will take longer to be updated and diffused. Another issue regarding performance is the contact time, which can have a strong influence especially in scenarios where contacts are short lived.

Another proposal known as Bubble Rap [16, 17] also uses node centrality along with the concept of community structure to perform forwarding. With Bubble Rap, nodes are grouped based on social parameters (i.e., number of contacts and contact duration) and have a local/global popularity index (obtained from betweenness centrality). Messages within the same community are forwarded using local popularity whereas messages traversing different communities use a combination of local/global popularity to reach the final destination. In the second case, whenever the message is forwarded to a member of the destination's community, the current carrier deletes it from the buffer to prevent further dissemination. The algorithm employed in this proposal is rather simple. If a source node wishes to send a message, all it needs to do is to check if the community of the destination node is the same as its own. If so, for every encountered intermediate node, it compares their local ranks and generates a copy to the encountered node that has higher rank value. Oth- 
erwise, it passes this message copy to an encountered node belonging to the same community as the destination or having higher global rank value.

Bubble Rap considers that nodes belong to different size communities and that such nodes have different levels of popularity (i.e., rank). With this, it can mimic human relationship allowing it to achieve good overall performance regarding delivery success rate with acceptable delivery cost. The performance is even better as the number of different communities and message TTL increase showing its capability to deal with human social behavior. However, reaching a destination in a different community is quite exhaustive, especially if the source node has the lowest rank and its community has a high number of nodes. This will incur undesirable replication within the source community. Moreover, centrality can result in overloading (e.g., processing, energy, buffer) popular nodes since they are outnumbered compared to the number of global nodes. In addition, it does not always mean that a high centrality node has the best contact probability with the destination community.

Differently from previous proposals, SocialCast [7] shows that forwarding can be achieved not only based on the social ties and mobility patterns, but also considering the interests of destinations. The proposed routing protocol determines a utility function based on the predicted node's co-location (i.e., probability of nodes being co-located with others sharing the same interest) and change in degree of connectivity (i.e., related to mobility and representing changes in neighbor sets), which is used to calculate how good data carrier a node can be. This proposal is based on the publish-subscribe paradigm, that is, nodes publish content on the network that is received by nodes according to their subscribed interests. Nodes get copies if they have higher utility regarding a given interest than the node currently carrying messages with content matching such interest. The proposal comprises three phases: i) interest dissemination, in which each node broadcasts, to its first-hop neighbors, its list of interests along with its updated utilities regarding its interests as well as the last received messages; ii) carrier selection, in which if the utility function of a neighbor node regarding a given interest is higher than the current carrier node, this neighbor is selected as the new carrier; and, iii) message dissemination, in which messages are replicated to interested nodes and/or passed to the new carrier.

SocialCast allows messages to reach their destinations with a very low number of replications (i.e., reduced resource consumption) and stable latency. The result is good delivery ratio with low TTL values as messages are delivered within few hops. However, the co-location assumption (i.e., nodes with same interests spend quite some time together) may not always be true [29]. Since such assumption is of great importance, the proposal is compromised in scenarios where it does not always apply.

Also considering node mobility and social interaction, PeopleRank [29] makes use of stable social information between nodes to decide on forwarding. As its own name suggests, PeopleRank sets ranks to nodes according to their social interaction, and use this ranking to decide on the next hop for data exchange as it is known that socially well-connected nodes become the best forwarders for message delivery. This ranking process is analogous to Google's page rank system in which the relative importance of a Web page is determined according to its links to/from a set of pages. 
Thus, nodes are ranked according to their position in the social graph, i.e., considering their linkage to other important nodes in the network. In order to determine its rank, a node needs to be acquainted to its socially connected neighbors and their respective ranks. So, when two nodes meet, they exchange their ranks and neighbor sets, update their own ranks, and exchange messages according to the new determined ranking. The node with the highest rank gets the messages.

The more social information is available to nodes, the better is the overall performance regarding delivery success rate. PeopleRank is also able to keep the cost associated to message delivery very low and with short delays. However, it is proven that considering only socially connected nodes is not enough to guarantee a good performance level, since socially disconnected nodes are also able to forward messages and could be considered to improve performance.

Most of the previous solutions focus solely inter-contact [6], and still significant investigation is required to understand the nature of such statistics (e.g., power-law, behavior dependent on node context) [21]. Another drawback of such approaches is the instable proximity graphs they create which follows mobility(encounter)-based social similarity metrics [17]. Instead of considering the dynamicity of mobility, the evolving feature of social network structure of nodes as they move around meeting different nodes throughout the day is what matters most. And such feature has been shown to be imperative when building proximity graphs based on social interactions [13].

Existing solutions [15, 8, 7, 17, 29] succeed in identifying similarities (e.g., affiliation, communities, interests) among users, but their performance is affected as dynamism derived from users' daily routines is not considered.

With dLife [28], the dynamism of users' behavior found in their daily life routines is considered to aid routing. The goal is to keep track of the different levels of social interactions (in terms of contact duration) nodes have throughout their daily activities in order to infer how well socially connected they are in different periods of the day.

The assumption here is that the time nodes spend together can be used as a measure of the strength of the social ties among them. To achieve that, dLife defines two utility functions: Time-Evolving Contact Duration (TECD) that measures the level of social interaction (i.e., social strength, $w(a, b)$ ) among pairs of nodes during their daily routine activities; and TECD Importance (TECDi) that measures the user's importance, $I(x)$, considering its node degree and the social strength towards its neighbors. Upon a contact, nodes exchange their social weights towards other nodes as well as their importance, and replication only occurs if the encountered node has a stronger social with the message's destination or higher importance in the system than the current message's carrier for that specific time period.

Despite of achieving good overall performance in terms of delivery probability and cost (with a delay increase trade-off), dLife does not reach maximum performance. It is believed that the introduction of some level of randomness may improve its performance, as randomness has been proved to increase delivery [29].

The Context Information Prediction for Routing in OppNets (CiPRO) [31] solution also takes advantages of what is happening in the daily routines of nodes. With 
CiPRO, the message carrier has enough knowledge about the time and place it will meet other nodes to forward the message. Such knowledge is defined in terms of node profile (with the carrier's - name, residence address, workplace, nationality, ... - and device's - battery level, memory, ... - information) which is used to compute encounter probability between a node and destination $p_{[N, D]}$ through a profile match. As in dLife, CiPRO's encounter probability can also reflect encounter happening in for specific time periods, $P_{i}$, which is given by a ratio between the sum of the probability of all the encountered nodes $E$ by the node towards the destination $D, p_{[E, D]}$, and the set of these nodes, $\left|N_{i}\right|$. When two nodes meet, CiPRO triggers a control message which is propagated up to its two-hop neighbors and contains the node profile information of the destination in a evidence/value pair format. Thus, it will forward messages according to the type of contact, namely occasional (where neighbors with higher encounter probability - computed based on the control message - towards the destination will get the message) and frequent (where the node will use the history of encounters to a given destination to foresee a specific time period for efficiently broadcasting control packets and the message itself). For the latter, a BackPropagation Neural Network model is used to determine the future encounter probability, $P_{\text {pred }}$.

Like dLife, CiPRO also has a performance trade-off between delivery probability/cost and delay, and perhaps introducing some randomness, in the occasional contact case, could bring more improvements to the solution.

In summary, proposals that take into consideration the idea of social similarity perform quite well when compared to algorithms simply based on history of encounters, encounter prediction, and message prioritization. However, most of the socialaware proposals consider the dynamism found in node mobility, instead of focusing on the dynamism of the social information that come from such movements. In addition, Hossmann et al. (2010) [13] show that, if the contact aggregation considered for determining the proximity graph is based on time window, some social metrics (e.g., betweenness centrality, similarity) can lead to node homogeneity regarding the given metric. That is, as network lifetime increases, these nodes may have the same characteristic (i.e., popularity) which will result in great impact on the performance of forwarding algorithms. This suggests that social-aware solutions must be carefully designed in order to not end up becoming a mere random-based solution. Moreover, most of the aforementioned proposals only supports point-to-point communication. (Multi)point-to-multipoint communication is a desirable feature in opportunistic routing as it can help reaching more nodes interested in the content of the messages with better performance and wise use of resources [41].

Now that we have learned about the social-aware solutions, we next present the existing taxonomies of opportunistic routing, identifying the appearance of the social-aware branch, and providing a simplified taxonomy proposed in $2011^{* *}$ with a minor update given the appearance of new social-aware opportunistic routing solutions. 


\section{Taxonomies}

The analysis of opportunistic routing approaches shows the existence of different trends based on distinct goals. On the one hand, we have single-copy forwarding approaches aiming to optimize the utilization of network resources. On the other hand, replication-based approaches aim to optimize delivery probability. Forwarding has the advantage of using network resources properly, but may end up taking too long to delivery the messages. Replication-based approaches present a message delivery probability that is, in almost every solution, very close to optimal, but with a high cost. Aiming to achieve a good balance between a high delivery probability and a low utilization of network resources, several proposals try to avoid flooding the network, by exploiting mobility of nodes, history of encounters, and social parameters.

Upon what is available in terms of opportunistic routing solutions, there are different classifications: considering aspects (e.g., level of knowledge) that led to an unbalanced classification, assigning most solutions to a few set of categories, or to very specific classification branches (e.g., by considering for instance information coding or methods to control movement of nodes). Independently of the classification approach used, our goal is to provide the reader with an overview of the existing opportunistic routing taxonomies in order to show when social similarity started to be considered and that its importance is recognized as it still appears evident in the latest taxonomies..

\subsection{Existing taxonomies}

The first taxonomy for opportunistic routing was proposed by Jain et al. (2004) [19] based on three types of classification. The most important one is the first type of classification, which divides opportunistic routing according to the knowledge about the network that nodes need to have to perform message forwarding. The second and third classifications follow a trend already used to classify other type of routing: the second approach classifies routing as proactive (i.e., route computation happens prior to traffic arrival) and reactive (i.e., route computation takes place upon the need for sending data); the third approach classifies routing as source-based (i.e., the complete route is determined by the source), or hop-based (i.e., the next hop is determined in every traversed hop).

In what concerns the knowledge-based taxonomy, knowledge about the network is provided by centralized oracles. Four different oracles are proposed: i) Contact Summary Oracle, which provides summarized information about contacts (e.g., average waiting time until next contact, average number of contacts); ii) Contacts Oracle, which provides more detailed information related to contacts between nodes at any point in time (e.g., number of contacts in a given period); iii) Queuing Oracle, which provides information about buffer utilization at any time; iv) Traffic Demand Oracle, which provides information about present or future traffic demand. Accord- 
ing to the authors, the more knowledge a routing solution can get, the better its performance will be, which leads to increasing unrealistic approaches since such ubiquitous knowledge is impossible to get in a dynamic network.

The knowledge levels used by each oracle can be: zero, where solutions use no information about the network to perform routing; partial, where solutions can route by using only the Contact Summary oracle, or one/both of the Contacts and Queuing oracles; and complete, where all oracles (Contacts, Queuing, and Traffic Demand) are considered. Fig. 2 illustrates all the different levels of knowledge used in this taxonomy as well as the existing oracles, and the relationship between performance and knowledge.

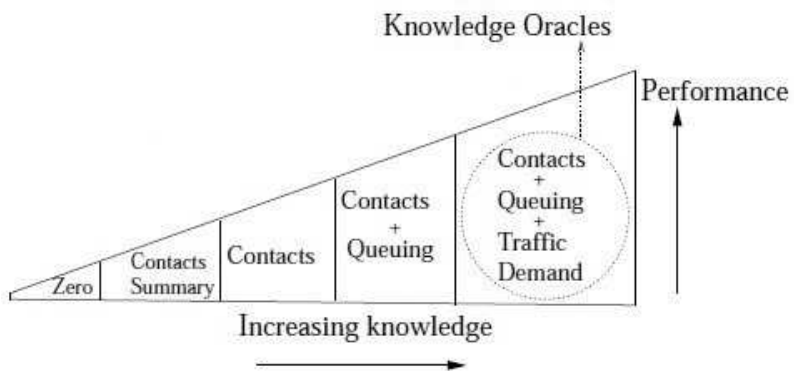

Fig. 2 Knowledge oracles (Jain et al. [19])

In realistic scenarios existing routing solutions are, however, classified as having zero or partial knowledge. This is due to the nature of opportunistic networks where network topology is not known beforehand, which makes it difficult to have a central entity (e.g., oracle) providing information to aid routing decisions. Not to mention the delay incurred to gather/process such information, which can become quite unfeasible in scenarios with short-lived contacts between nodes. Regarding the time and place for routing decisions, most of the solutions can be seen as hop-by-hop reactive approaches.

In our opinion, a taxonomy based on network knowledge, as well as proactive/reactive and source/hop characteristics is not the most suitable one to provide a balanced classification of opportunistic routing approaches.

The most complete taxonomy for opportunistic routing, to our knowledge, is provided by Zhang (2006) [40]. This taxonomy follows the work presented by Jain et al., classifying protocols according to the information they require from network as well as relevant routing strategies (e.g., pure forwarding, estimation of forwarding probability). The result is a taxonomy based on two categories, namely deterministic and stochastic routing. In the former case, node movement and future connections are known beforehand (i.e., nodes are completely aware about topology), which follows the oracle-based taxonomy proposed by Jain et al. In the case of stochastic approaches, the behavior of nodes and network is random and unknown. Hence, routing decisions depend upon local conditions leading to simple solutions where 
messages are replicated on every contact up to more complex solutions where the use of history of encounters, node mobility patterns, and message coding are considered for routing decisions. Zhang also classifies proposals regarding whether (or not) node movement can be controlled.

We can say that Zhang's proposal complements the proposal of Jain et al. by including a set of more realistic (stochastic) approaches. However, Zhang's proposal emphasizes aspects that are orthogonal to different routing categories (i.e., coding methods) and puts emphasis on categories that are specific to deterministic networks (e.g., node movement control), which do not represent a scenario where opportunistic routing may have greater impact in our daily life.

Balasubramanian et al. (2007) [1] propose a taxonomy based on two types of classification criteria regarding the routing strategy and the effect on performance metrics. Based on the first criterion, solutions are divided into two routing strategies: replication-based, where messages are replicated and then transferred to the next hop; and, ii) forwarding-based, where only one copy of the message traverses the network. The second classification criterion is used to divide solutions based on the effect routing decisions have on performance metrics. There are two types of effect: i) incidental, where the effect of decisions do not take into consideration resource constraints; and, ii) intentional, which determines the effect of a routing strategy on metrics considering such constraints.

We believe that classifying routing solutions according to their routing strategy is the most suitable approach. However, there is more to it than what is presented by Balasubramanian et al. since there are other performance metrics, besides incidental/intentional usage of resources, that can be used to distinguish among solutions that follow the same generic strategy (i.e., replication).

The same idea of classifying solutions based on their routing strategy (i.e., forwarding/replication) is also followed by Song and Kotz (2007) [34] and Nelson et al. (2009) [30]. In relation to the work presented by Balasubramanian et al., the classification presented by Song and Kotz is able to further divide replication-based proposals taking into consideration, not only the effect that they have on network resource consumption, but also on delivery probability. Still in what concerns the classification of replication-based approaches, Nelson et al. propose to divide them into flooding-based and quota-based. This classification is quite important since its shows that it is not the fact that messages are duplicated that may lead to a flooding situation, as the one that occurs with epidemic routing, but the employed routing metric for deciding on replication. Nelson et al. shows that there are probabilisticbased replication strategies that actually end up flooding the network (floodingbased), since at the end of the experimental period every node has at least one replica of each message, while others (quota-based) have higher success in controlling the number of replicas in the network. However, this taxonomy is incomplete in the sense that it does not consider routing categories based on metrics such as encounter number and resource usage.

In another survey [9], D'Souza and Jose (2010) classify routing solutions into three major categories: i) flooding-based, where nodes flood the network to increase delivery probability or apply some measures to control such flooding by bounding 
the number of messages copies to be distributed in the network and by embedding additional information to messages blocks to reduce flooding effects; ii) historybased, in which the history of encounters between nodes is taken into account to improve routing decisions; and, iii) special devices-based, where stationary or mobile devices are used to improve communication among the communicating nodes. Such special devices can also consider social interaction among nodes to perform routing decisions. Like Zhang, D'Souza and Jose consider aspects that are orthogonal (e.g., network and erasure coding) and could be easily applied to other categories. Despite the fact that the first social-aware solution appeared in 2007 (i.e., Label [15]), such category of opportunistic routing only made it to taxonomies in 2010 with D'Souza and Jose's work. Still, their taxonomy proposal includes this trend under a category (i.e., special devices-based) that does not comply with the regular behavior (i.e., random and unknown) found in opportunistic networks. We believe that distinguishing proposals according to whether or not they use special stationary/mobile devices to improve data exchange is not realistic as the network/nodes will have to present a deterministic behavior in order to correctly place these devices in the system.

The classification proposed by Spyropoulos et al. (2010) [38] groups opportunistic routing proposals according to message exchange scheme they employ: forwarding (only one message copy traverse the network), replication (message is replicated in different levels ranging from every node getting a copy up to more elaborate solutions based on utility functions), and coding (where message can be coded and processed at the source or as it travels throughout the network). The authors also identify the different types of utility functions that can be applied to either forwarding or replication message schemes. Such functions are categorized according to their dependency on the destination (i.e., destination dependent/independent). Additionally, they classify DTNs according to characteristics that have major impact on routing such as connectivity, mobility, node resources, and application requirements. The authors succeed in mapping the routing solutions to the different types of DTNs. Still, the proposed opportunistic routing classification considers categories that can be orthogonal (i.e., coding) and does not include the social similarity trend observed in 2007. They simply refer to the social aspects as a mere destinationdependent function in which we believe comprises a new research direction that includes social relationships, interests, and popularity to achieve suitable delivery probability with shorter delay and cost.

Fig. 3 summarizes the analyzed taxonomies. Such figure shows an evolution towards stochastic approaches that do not require any knowledge about the global network topology. Deterministic approaches, based on some kind of centralized oracles, are not realistic and do not reflect the behavior found in opportunistic networks. Within stochastic approaches, and since 2007, there is a clear trend to classify routing strategies considering their success in achieving a good balance between delivery probability (e.g., message replication) and usage of network resources (e.g., forwarding) by employing the social similarity approach.

Keeping in mind the ultimate goal of balancing performance and resource usage, current taxonomies are not capable of illustrating the different families of routing metrics that have been used to devise replication-based approaches able to avoid 


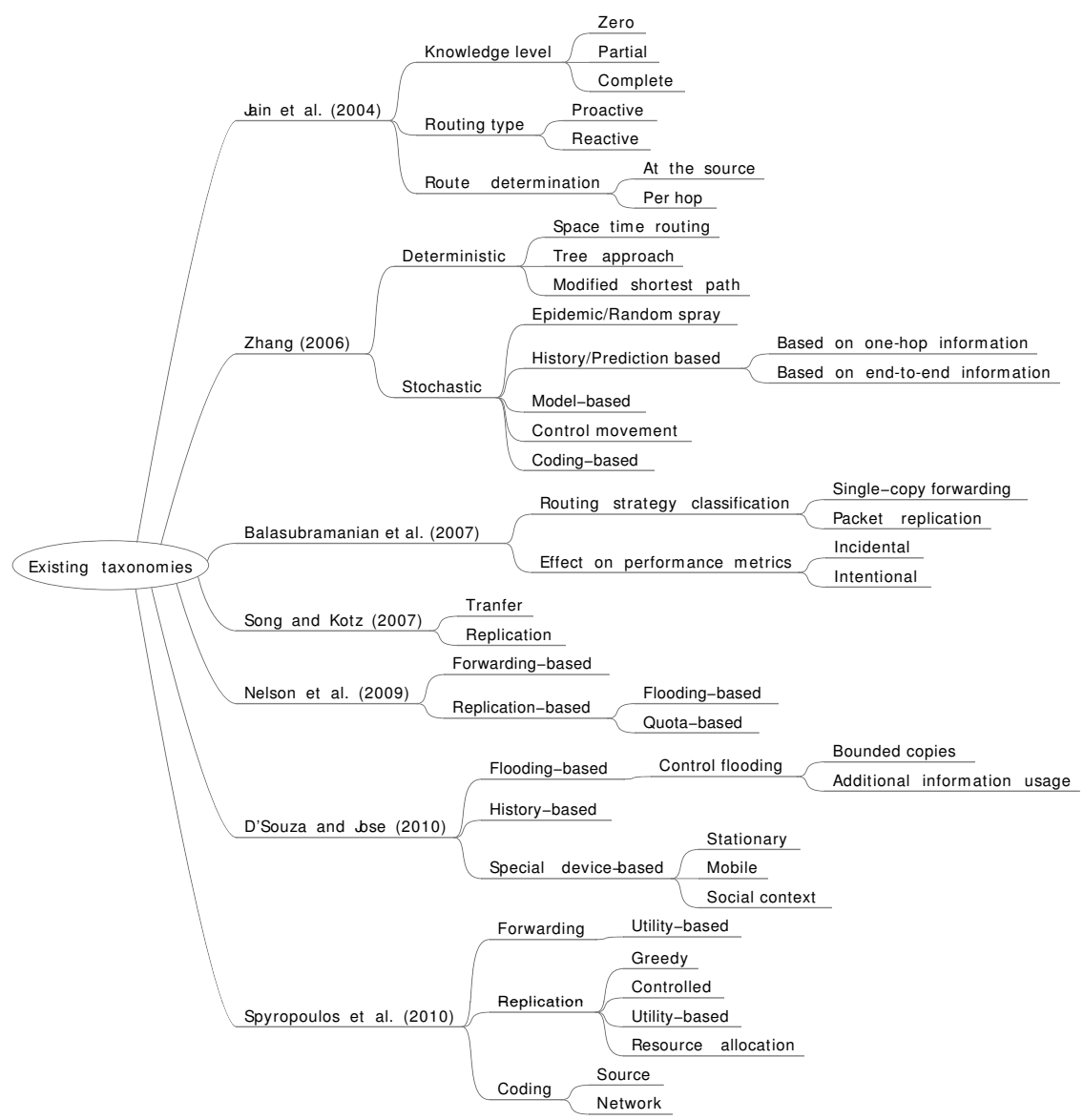

Fig. 3 Existing taxonomies

network flooding. Next, we present a simplified proposal to extend the taxonomy (presented by Nelson et al.) that most reflects the behavior of opportunistic networks with a set of categories that represent recent trends in stochastic opportunistic routing.

\subsection{Proposed Taxonomy}

One can conclude that the existing taxonomies generally focus on the analysis of opportunistic routing proposals based on their efficiency (e.g., level of knowledge employed to achieve higher delivery rates [19], forwarding schemes that result in different performance levels [40, 1, 34], limiting the number of messages copies in the network to spare resources [30, 9], or application of the correct routing algorithm 
according to the specificity of the network [38]), but they lack an analysis of the characteristics of the graph structure used.

We believe that focusing on an analysis of the topological features (e.g., contact frequency and age, resource utilization, community formation, common interests, node popularity) assumed by each proposal may lead to a more stable taxonomy useful to study real-world networks such as computer networks operated based on social behavior.

Thus, in this section, we present a 2011 taxonomy including updates based on the emergence of recent opportunistic routing proposals (cf. section 3).

Social similarity is an example of recent metrics that have clearly created a new trend in the investigation of opportunistic routing [15, 8, 7, 16, 29, 28, 31]. The reason for this is that social behavior takes into account human relationship characteristics such as contacts with other people, time spent with these people, the level of relationship between people, among others. And, since computing devices are carried by humans, social-based forwarding decisions can consider people's socially meaningful relationships, where the relevant information come from aspects such as human mobility, interaction and social structures. This information can be used to perform forwarding, because the topology created from human social behavior varies less than the one based on mobility and thus such solutions deserve being categorized.

It is important to mention that all studied opportunistic routing proposals take advantage of node mobility to forward data ahead, where some of them (e.g., Epidemic [39], Direct Transmission [37]) are rather simple and use the resulting contacts to reach the destination, while others are more elaborate and consider social aspects in order to find the destination (e.g., LABEL [15], PeopleRank [29]). This is the reason why Moreira et al. (2011) do not devote a specific category (as Model-or Control Movement-based in Zhang [40] and Mobile Device-based in D'Souza and Jose [9]) since this is an inherent feature of opportunistic proposals and instead look for features that help understanding their graph structure.

The proposed taxonomy is based on an initial classification of all proposals as forwarding-, flooding-, or replication-based. The forwarding-based category is also known as single-copy forwarding (e.g., MEED, and approaches in Spyropoulos et al. [37]) since all approaches propose that only one copy of each message traverses the network towards the destination. From the resource consumption viewpoint, this category of approaches is quite interesting since it keeps network (e.g., bandwidth) and node (e.g., buffer space) resources usage at a low level; however, all approaches suffers in general from high delay rates that, consequently, results in a low delivery rate.

Nelson et al. propose, depending on the level of duplication, that algorithms within the replication-based approach be divided into flooding-based and quotabased. The flooding-based algorithms are able to increase delivery rate to a very high level, whereas the quota-based algorithms, in general, allow a more wise usage of resources, resulting in low delay and reduced flooding overhead since they tend to spread less copies of messages in the network. 
Moreira et al. do consider these different levels of replication but unlike Nelson et al., it is proposed that flooding-based algorithms be classified out of the replication branch. First, because only proposals that allow every node to spread a copy of each message to every other node that they meet (e.g., Epidemic) are considered. And, also due to the fact that having (or not) the quota-based feature (i.e., where the number of created copies does not depend on the number of network nodes) can be found in the different algorithms identified in Section 2

Despite being an aggressive approach, the flooding-based strategy is able to increase delivery rate, but at the same time leads to a high consumption of resources. Such waste of resources can be avoided with algorithms that try to somehow control flooding. This control starts by limiting the number of copies injected in the network, if it is able to avoid nodes ending up with a replica of every created message, the algorithm has the quota-based feature.

So, the replication-based approaches have as common goal an attempt to increase the delivery rate by sending several copies of the initial message through different nodes to quickly reach the destination before message expiration time. Since these approaches consider different routing algorithms and metrics, these are its sub-categories.

The first sub-category is the encounter-based, where nodes choose next hops based either on frequency encounters (e.g., PROPHET, MaxProp, and Prediction, and EBR), or aging encounters (e.g., FRESH, EASE, and Spray and Focus). In the former, proposals consider the history of encounters with a specific destination to support opportunistic forwarding of messages or the frequency nodes met in the past to predict future encounters. As for the latter, proposals consider the time elapsed since the last encounter with the destination to decide about next hops.

Resource usage is the second sub-category, in which decisions are made considering the age of messages (e.g., Spray and Wait, and $O P F$ ) or knowledge about local resources (e.g., PREP, and RAPID). Aging messages proposals have in common the fact that they aim to avoid messages to be kept being forwarded in the network by creating metrics that define the age of message copies. As for the resource allocation proposals, they take forwarding decisions that wisely use available resources.

The last sub-category is related to social similarity, where proposals start to follow more complex algorithms aiming first at avoiding flooding with high probability, and exploiting social behavior. Thus, social similarity algorithms are divided into: community detection, shared interest, and node popularity.

Community detection approaches (e.g., SimBet, Label, BubbleRap) rely on the creation of node communities taking into consideration people social relationships translated to contact numbers and duration of contact among nodes. These approaches suffer with the overhead of community formation. The shared interest approach (e.g., SocialCast) relies on the assumption that nodes with the same interest as the destination of the message are good forwarders since they have high probability to meet. But this assumption may not always be true [29], since a node with similar interest to a given group may not even come in contact with this group of nodes. Still within the social similarity category, there are approaches that are based only on a process of ranking people in terms of their popularity without a straight 
dependency upon neither the computation of communities nor the synchronization of interests. Node popularity approaches (e.g., PeopleRank) make use of social information to generate ranks to nodes based on their position on a social graph, using such ranking to decide on the next hop for data exchange. Although social similarity algorithms provide stable graphs, it is proven that relying on socially connected nodes may not be enough to guarantee a good performance, which can be improved with the inclusion of some degree of randomness in the forwarding decision [29].

With the appearance of opportunistic routing solutions which take into account the dynamism of user social behavior (i.e., $d$ Life and $C i P R O$ ), we propose an update

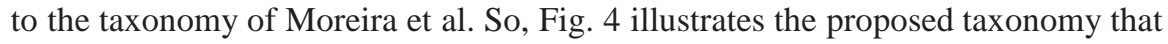
complements the most recent trend (replication vs. forwarding) with the analysis done of twenty-two proposals published between 2000 and 2012 and includes the new sub-category, user dynamic behavior.

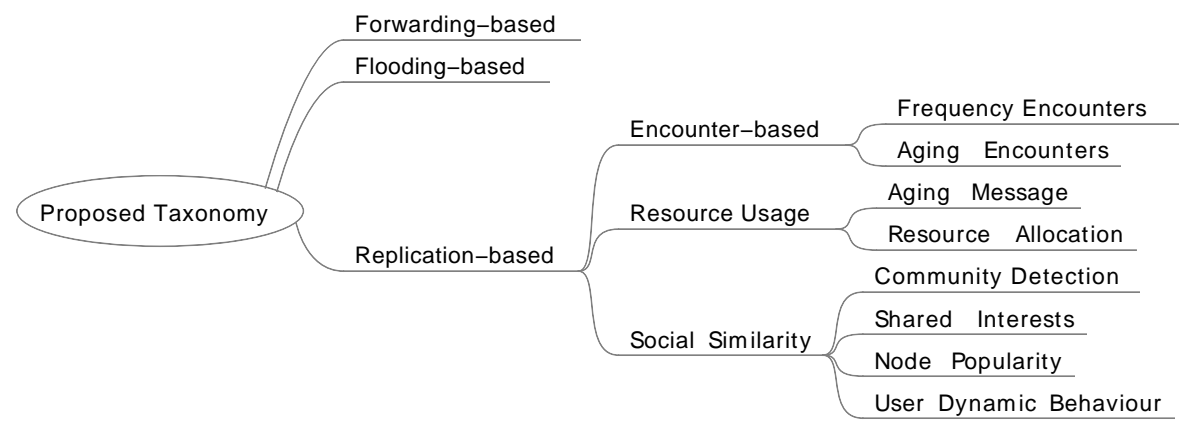

Fig. 4 Taxonomy for opportunistic routing

It is easily observed that all categories of the presented taxonomy has advantages and disadvantages. However, our goal was not to identify the winner category, but to: i) present the reader with reasons to support the emergence of the new trend based on social similarity; and ii) update a previously proposed taxonomy with a new sub-category based on the latest social-aware opportunistic routing proposals.

\section{Experiments}

We start this section by describing the employed evaluation methodology and simulation settings. Then, we present our considerations on the obtained results pointing out the advantages and constraints of using social similarity existing among nodes to perform forwarding in opportunistic networks.

The results are presented in two parts: first, we show the results in a scenario with synthetic mobility models (hereafter referred to as heterogeneous scenario); then, we present a performance comparison between some of the aforementioned 
opportunistic routing proposals to support our case: social similarity is indeed a trend and has great potential in improving opportunistic routing.

\subsection{Evaluation Methodology}

Our experiments are done based on the Opportunistic Network Environment (ONE) simulator [22].

For that, we run simulations representing a 12-day interaction period (with 2 days of warmup, not considered for the results) in our heterogeneous scenario. For the human trace-based simulations, we considered the Cambridge traces [33]. Each simulation is run ten times (with different random number generator seeds for the used movement models) in order to provide results with a 95\% confidence interval. All results are analyzed considering the average delivery probability (i.e., ratio between the number of delivered messages and total number of created messages), average cost (i.e., number of replicas per delivered message), and average latency (i.e., time elapsed between message creation and delivery).

Regarding the ONE simulator, the time step size is of 2 seconds for the heterogeneous scenario and of 1 second for the trace-based scenario for more precision in the collected data. All simulations are performed in batch mode with 2 GB RAM dedicated memory.

\subsection{Simulation Settings}

With our heterogeneous scenario simulation settings, we aimed at creating a scenario that is as close as possible to the heterogeneous environment (in terms of mobility) that people find in their daily activities in a city, such as pedestrian walks, combined with bus and car rides, as well as people attraction to diverse locations such as work, shopping places and home.

Our simulation scenario is part of the Helsinki city (available in ONE) and has 150 nodes distributed in 17 groups ( 8 groups of people and 9 groups of vehicles). All nodes are equipped with one WiFi interface $(11 \mathrm{Mbps} / 100 \mathrm{~m})$. One of the vehicle groups, with 10 nodes, follows the Shortest Path Map Based Movement (SPMBM) mobility model, based on which nodes randomly choose a point in the map and use the shortest path to reach it. These nodes represent for instance police patrols. They move with speed between 7 to $10 \mathrm{~m} / \mathrm{s}$ and have a waiting time between 100 and 300 seconds when arriving at the chosen destination.

The other eight vehicle groups represent buses that cover different parts of the city. Each group is composed of 2 vehicles each. They follow the Bus Movement

mobility model with speeds between 7 to $10 \mathrm{~m} / \mathrm{s}$ and have waiting times between 10 and 30 seconds. 
Regarding groups of people, they follow the Working Day Movement (WDM) mobility model with walking speeds ranging from 0.8 to $1.4 \mathrm{~m} / \mathrm{s}$. People may also use buses to move around the city. Each group has different meeting spots, offices, and home locations. People spend 8 hours at work and present $50 \%$ probability of having an evening activity after leaving work. In the office, nodes move around and have a pause time ranging from 1 minute to 4 hours. Evening activities can be done alone or in a group, with a maximum of 3 people each. Each evening activity can last between 1 and 2 hours.

For the human trace-based simulations, the Cambridge trace was considered which corresponds to a 2-month imote communication between 36 students carrying these devices throughout their daily activities.

The traffic load used in the simulations comes from a file previously generated, as has established source/destination pairs, where approximately 500 messages are generated per day among a subset of node pairs. In a simulation of 12 days (i.e., heterogeneous scenario), with 2 days of warmup time, this results in a total of 6000 messages, from which 5018 messages are considered for the performance assessment. As for the trace-based simulations, all the 6000 messages are considered in the assessment.

Message TTL values are set at either 1,2 and 4 days, as well as 1 and 3 weeks. Since we want to bring our experiments as close as possible to the real world, we chose values that can represent the different applications which cope with opportunistic routing. Message size ranges from $1 \mathrm{kB}$ to $100 \mathrm{kB}$. The buffer space is of $2 \mathrm{MB}$ as users may not be willing to share all of their storage space. Message and buffer size comply with the universal evaluation framework that we proposed previously [25, 26, 27] based on the evidence that prior-art on opportunistic routing (19 proposals from 2000 to 2010) follows completely different evaluation settings, making the assessment a challenging task.

The proposals considered for our experiments comprise social-oblivious as well as social aware solutions. As representative of the former class of opportunistic routing, we chose Epidemic [39] (normally serves as upper bound for delivery probability), PROPHET v1 [23] (accepted as standard in by the Delay Tolerant Networks research community), and Spray and Wait [35] (appearing as lower bound for latency) for being the most cited proposals (i.e., often used as benchmark for performance comparison) [25]. For the social-aware approaches, Bubble Rap [17], dLife and dLifecomm [28] were selected as representative of solutions considering social structures (i.e., communities), node popularity, and dynamic behavior of users.

Some of the simulated proposals need to have some parameters set, thus we set: i) PROPHET with aging of delivery predictability happening at every 30 seconds; ii) Spray and Wait, being binary and with a number of spraying copy set to 10; iii) dLifeComm and Bubble Rap, with K-Clique ( $k=5,700$-second familiar threshold) and cumulative window algorithms for community formation and node centrality computation; and iv) dLife and dLifeComm, with 24 daily samples. The choice for such values are among those which each proposal has presented good overall performance as specified in their respective original papers.

Table1 1 summarizes the setup parameters. 
Table 1 Simulation parameters

\begin{tabular}{lll}
\hline Parameters & Values & \\
\hline Simulator & Opportunistic Network Environment (ONE) & \\
Routing Proposals & Epidemic, PROPHET, Spray\&Wait, Bubble Rap, dLife and dLifeComm \\
Scenarios & Heterogeneous & Trace Cambridge \\
Simulation Time & $1036800 \mathrm{sec}$ & $1000000 \mathrm{sec}$ \\
\# of nodes & 150 (people/vehicles) & 36 (people) \\
Mobility Models & Working Day, Bus, Shortest Path Map Based & Human \\
Node Interface & Wi-Fi (Rate: 11 Mbps / Range: 100 m) & Bluetooth \\
Node Buffer & $2 \mathrm{MB}$ & \\
Message TTL & $1,2,4$ days, 1 and 3 weeks & \\
Message Size & $1-100 \mathrm{kB}$ & \\
\# of Messages & 6000 (only 5018 considered for the heterogeneous scenario) \\
Spraying copies & $\mathrm{L}=10$ (Spray and Wait) \\
K-Clique & $\mathrm{k}=5$ and familiarThreshold = 700 seconds (Bubble Rap and dLifeComm) \\
Daily Samples & 24 (dLife and dLifeComm) \\
\hline
\end{tabular}

It is important to say that our goal is not to show which proposal is the best. Instead, we want the reader to understand the potential of social-aware opportunistic solutions and why they haven shown to be a trend in the last years for opportunistic networks.

Next we present the experiments results.

\subsection{Results}

Before we present the results of our experiments, here are a few general observations regarding our findings. The average number of contacts per hour is of approximately 962 in the heterogeneous scenario and of 32 in the trace-based one. Additionally, contacts are more sporadic in the trace-based scenario than in the heterogeneous one, in which contact frequency is more homogeneous. We also observe that the average number of unique communities is higher in the heterogeneous scenario $(\sim 68)$ than in the trace-based scenario $(\sim 8.7)$. Furthermore, most of the created communities encompasses all the existing nodes (150 for the heterogeneous simulations, and 36 for trace), which means that independently of the level of contact homogeneity, nodes are still well connected. Heterogeneous scenario

Fig. 5 presents the results for the average delivery probability. It is clear that Epidemic (normally seen as an upper bound for this performance metric) has the worst performance amongst the proposals. This is due to the available buffer space (2 MB) which was reduced as to represent a limited willingness of the user to carry information of behalf of others.

As we could see [25, 26, 27], authors tend to consider unlimited storage. However, while this may be true in scenarios comprising nodes for the serving purpose 
(e.g., [1]), normally a user may find him/herself in an opportunistic network formed on-the-fly and surrounded by other users sharing storage space according to their devices' capabilities and mostly to their willingness in cooperating.

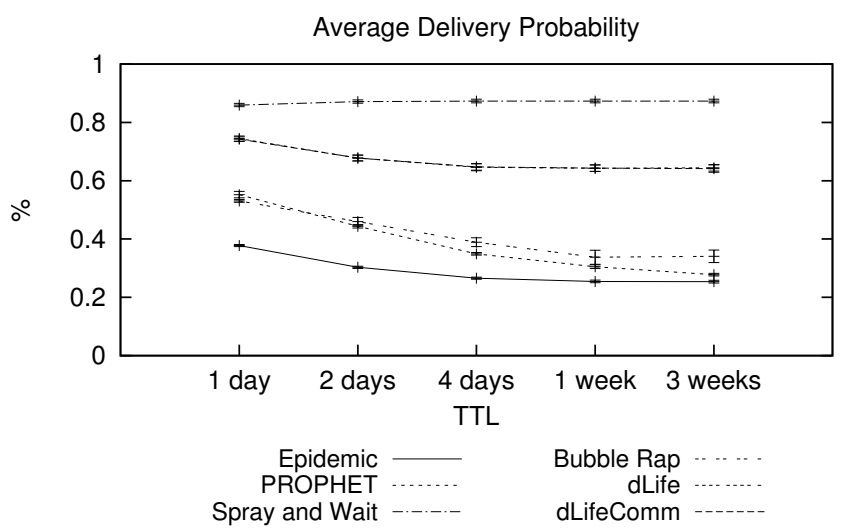

Fig. 5 Average delivery probability

PROPHET and Bubble Rap are also affected by the limited buffer and their delivery capability diminishes as TTL increases. Since messages are allowed longer in the network (i.e., being replicated), this consequently results in buffer exhaustion. We believe that the mobility heterogeneity found in the scenario also contributes for the decrease in the delivery probability.

Since the dynamic behavior of users are considered, both dLife and dLifeComm are less affected by the limited buffer space. They carefully decide whether to replicate based on the social weight towards the destination and node importance in the network at the time of the encounter. Thus, wiser decisions take place as next forwarders are only chosen if they indeed have a stronger social connection or importance than the current message carriers.

Spray and Wait has the best performance. This is due to the fact that the scenario comprises nodes (e.g., buses and police patrols) which cover most of the simulated area and this proposal takes advantage of that. Most of its random replications happen to be to such nodes and as they move across the entire scenario added to the 100-meter transmission range, its delivery capability increases. Despite the better performance, Spray and Wait still does not take advantage of longer TTLs to reach optimum delivery as some of the messages end up in the possession of nodes which are not well socially connected to their destinations.

Fig. 6] shows the performance of each proposal considering the number of replicas created per delivered message. As one could expect, Epidemic and Spray and Wait are the upper and lower bounds for this metric. The former has the highest cost as it replicates a message with every encountered node which does not have a copy yet. The scenario itself is not epidemic-friendly since it has a high number of contacts among nodes. On the other hand, Spray and Wait is allowed to create up 
to ten $(L=10)$ copies per messages, and thus its cost is the lowest (average across simulations of $\sim 10.16$ replicas per delivery). It is important to note that the way the ONE simulator determines cost (a.k.a. overhead) is given by the ratio between the number of successful relayed message (discarding the relaying to the final destination, i.e., number of delivered messages) and number of delivered messages. This explains why cost here exceeds the one $L$ specifies for Spray and Wait.

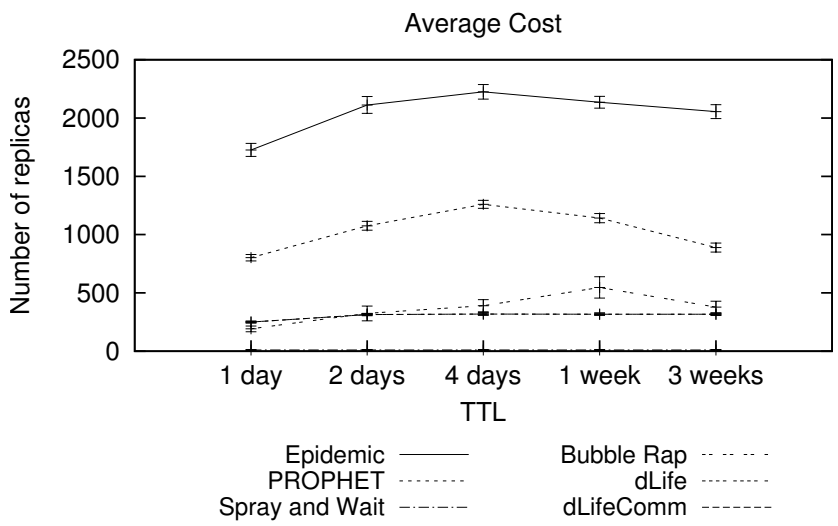

Fig. 6 Average cost

By being a probabilistic-based solution, PROPHET considers the frequency of past contacts with the message's destination to decide on replication. Since the proposal ages such encounters, only nodes that have frequent contacts with the destination will be entitled to receive a copy. Despite the effort of the proposal, it still requires a high number of replicas to perform a successful delivery.

Bubble Rap, dLife and dLifeComm have a much lower cost when compared to Epidemic and PROPHET, which shows that taking forwarding decisions based on some level of social similarity is beneficial as messages are replicated to nodes well socially connected to the destination of message. Regarding Bubble Rap, its cost is expected to increase as TTL increases [16, 17]; however, there is still a drop in cost observed for a 3-week TTL. We believe this is due to the fact that the proposal has taken advantage of the longer TTL, choosing next forwarders much more wisely, which reduces its cost. As a matter of fact, this can be confirmed in Fig. 5 where the same performance is achieved but with much less replications.

Both dLife and dLifeComm present a much more stable behavior as they consider the dynamism found in the users' daily routines and can choose to replicate only to nodes that actually have the best social interactions with the message's destination at each encounter.

A Fig. 7 shows the performance of the proposals in terms of average latency for delivering messages. As mentioned earlier, Spray and Wait takes advantage of the scenario which has nodes (i.e., buses and police patrols) covering the whole extension of the scenario and with longer transmission range. This significantly reduces 
this proposal's latency as destinations can be reached in shorter periods of time as reported in its original paper [35]. Epidemic also has short latencies as spreading many copies of the same message also reduces the time it will take to reach its destination.

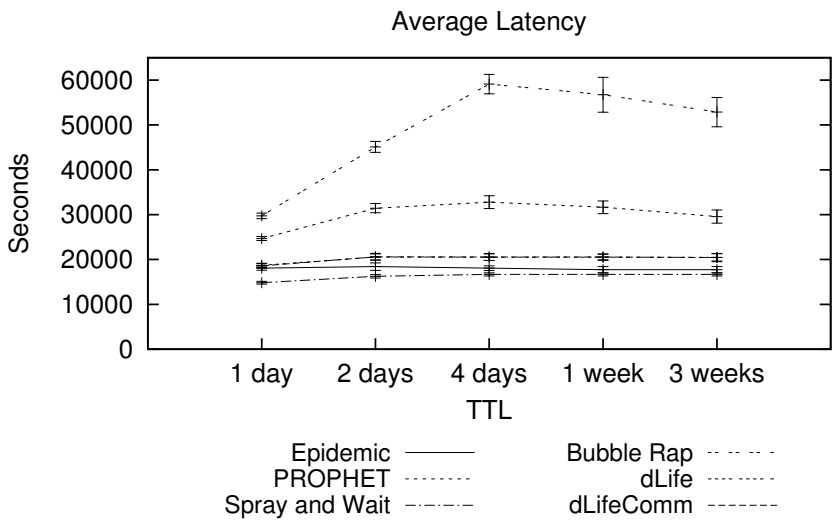

Fig. 7 Average latency

As waiting for the best (i.e., socially speaking) next forwarder may take some time, dLife and dLifeComm have a slight increase (varying between 490 and 4350 seconds) in latency when compared to both Epidemic and Spray and Wait. Thus, one can observe the tradeoff when only forwarding messages in the presence of strong social links or highly important nodes in the current daily sample. Still, despite of taking a little longer reach their destinations, dLife and dLifeComm are able to deliver a reasonable amount of messages.

PROPHET does not consider the social strength of the next forwarder with the destination; instead, it looks at the frequency of past interactions and this results in replication of messages that may take longer to reach their destinations. This can also be confirmed with the performance of Bubble Rap regarding this metric: it chooses also to replicate according to the centrality (i.e., cumulative number of unique contacts within 6-hour time windows) of nodes. Centrality indeed identifies nodes which may be well connected throughout the the entire experiments, but fails to capture the different levels of "being socially connected)" during the different periods of the day. To make matters worst given the number of average contacts per hour in this scenario (962), this proposal rely solely on global centrality while communities are forming. The result is replicas being created to nodes with weak social ties to destinations and which account for the total average latency experienced to deliver messages.

After looking at the performance of the proposals in a scenario of synthetic mobility models, we next present the performance of the same proposals using real human traces. With this, we expect to have experiments which are as close as possible from the reality when it comes to human mobility. 


\subsubsection{Cambridge scenario}

Fig. 8 presents the performance of the considered proposal in terms of average delivery probability. One can easily see that the advantage of Spray and Wait seen in the previous scenario has decreased. This is mainly due to the fact that in this scenario there are no nodes covering the whole area. Instead, nodes are encountering others as they move throughout their daily routines. Additionally, contacts are much more sporadic. Under these circumstances, Spray and Wait spreads copies to nodes that may never come in contact with the destination, thus reducing its delivery capability.

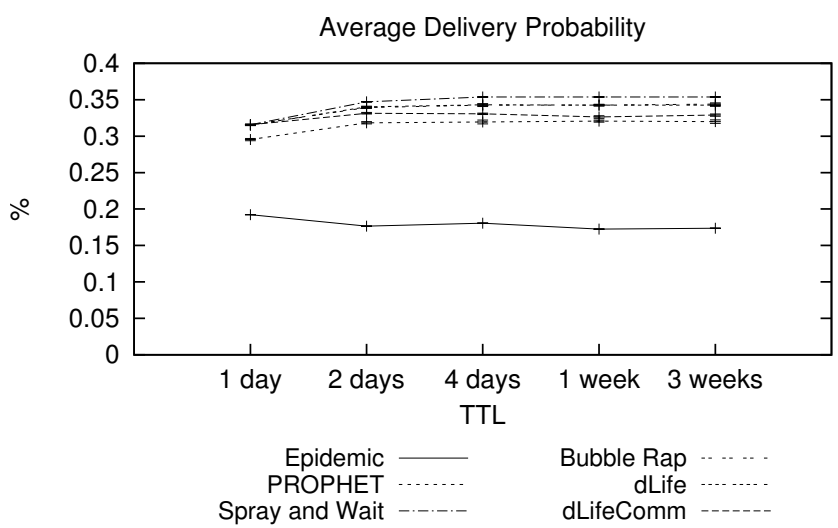

Fig. 8 Average delivery probability

This contact sporadicity also affects the remaining proposals since the solutions somehow depend on how nodes interact among themselves. Creating communities and determining centrality and importance of nodes as well as social weight among them take much longer in a scenario with low number of contacts ( 32 per hour). This is why dLife and Bubble Rap are statistically equivalent and perform very much the same. dLifeComm relies on the node importance to replicate, as node importance is more elaborate and will take longer to distinguish really important nodes, message will be replicated to nodes not well socially related with the destination. Despite being based in the notion of community formation, Bubble Rap's centrality is simple and can distinguish nodes much easier.

PROPHET faces the same issue, delivery predictabilities of nodes are not well computed and nodes getting copies are not the best option to increase its delivery capacity. And despite of the uncontrolled replication, Epidemic does not reach its best in this scenario as nodes meet occasionally added to limited buffer.

In Fig. 9 we can see also the effect of the sporadic contacts. The number of copies to perform a delivery is much less since there are only few nodes to receive such copies at the time of exchange. Still Epidemic is the proposal that most replicates (despite of a decrease varying between 877 and 1300 replicas per delivery) and Spray and Wait remains the proposal with the least cost, as one could already ex- 
pect; however, this time with a little increase (average across simulations of $~ 15.12$ replicas per delivery).

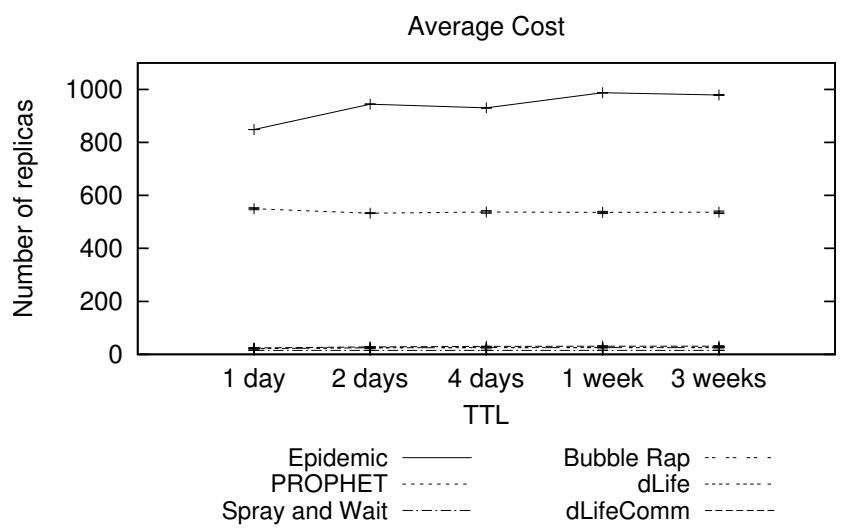

Fig. 9 Average cost

PROPHET experienced reductions varying between 252 and 606 replicas per delivery and remains as the second proposal to consume more resources. The socialaware solutions, namely Bubble Rap, dLife and dLifeComm, managed to have an average across simulations of approximately $24.52,24.56$, and 28.79 replicas per delivery. Here we can observe the potential of social-aware opportunistic routing as with a few extra copies they can almost reach the same delivery of the socialoblivious Spray and Wait proposal.

As contacts are rather sporadic, one could expect an increase in latency when delivering messages in the trace-based scenario. Indeed, this can be observed in Fig. 10 where all the proposals took longer times to perform deliveries when compared to the heterogeneous scenario. To influence even more in the time to deliver messages, we observe an increase in the distance (i.e., average number of hops of at most 2) to reach the destination in the trace-based simulations. This means that, besides the time taken to decide whether or not to replicate, delivery also accounts for the time messages traverse different hops. The consequence is that for all cases, proposals almost doubled their time to deliver messages. 


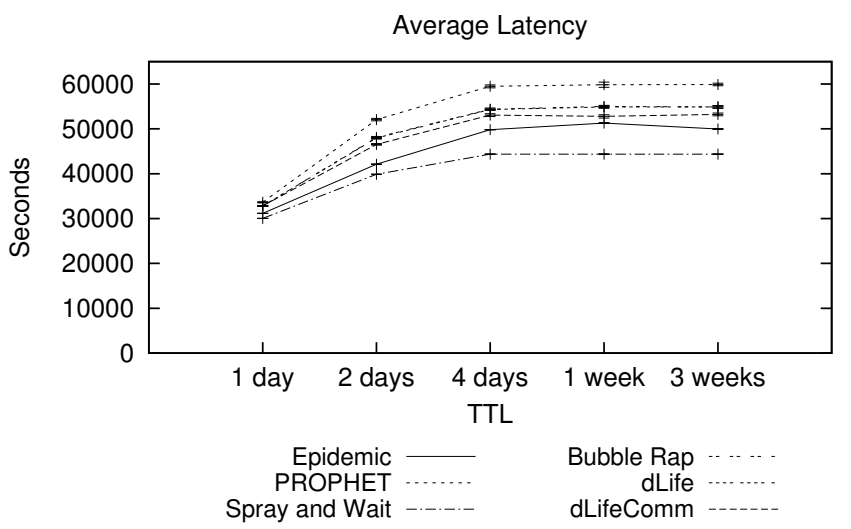

Fig. 10 Average latency

We believe this is due to the fact that most of the nodes encounter one another (although in a sporadic manner) through the simulations, which leads proposals to not have information reliable enough to suitably decide on replication. Thus, an increase in the distance to reach destinations.

Amongst the proposals Bubble Rap was the proposal that least experienced an increase in latency, having an almost similar behavior to the one observed in the heterogeneous scenario. As a matter of fact, this proposal experienced a decrease in latency with TTL of 4 days (less 4709 seconds) and 1 week (less 1725 seconds). This scenario has not been favorable for PROPHET (with the highest latency values), as contact sporadicity really affects the notion of time of last encounter employ by the proposal. dLife and dLifeComm were affected in the sense that the scenario is not dynamic given the non-homogeneous frequency of contacts among nodes. Since it spreads many copies, Epidemic still keeps its position of second best solution in terms of latency as one of these many replicas will reach the destination in short times. And Spray and Wait has the overall latency when it comes to delivering messages in shorter times.

\subsubsection{Considerations}

A subset of social-oblivious and social aware solutions are evaluated under the same conditions and considering two different scenario. Each of these scenarios have particularities and specific challenges. In the first, heterogeneous scenario, nodes follow different mobility models (representing people, buses and police patrols) and interact with one another as the move between home and work, go to a leisure activity, transport people and secure the city. This is a scenario with a high numbers of contacts among nodes where social similarity can be easily inferred as they move throughout their daily routines.

We could observe that this scenario is advantageous to the social-oblivious Spray and Wait as it comprises nodes that cover most of the simulated area and by ran- 
domly spraying copies to such nodes, Spray and Wait manages to fast deliver the highest number of messages ( 86\%) with the lowest cost (at most 10.12 copies to perform a delivery). Still, the social-aware solutions, $d$ Life and $d L i f e C o m m$, are able to capture the dynamicity of user behavior and with a small latency tradeoff (25\% increase when compared to Spray and Wait) they reach up to $74 \%$ delivery with a wise usage of resources (i.e., buffer space) producing at most 319 replicas to perform a successful delivery.

Since user dynamicity is not considered, Bubble Rap suffers from the problem of forming communities: as they are not readily available, the proposal will solely rely on centrality to replicate information, which also take some time to represent the system's reality. This results in a delivery probability not more than $53 \%$ with cost reaching up to $\sim 547$ replicas to successfully deliver messages and latency increase up to $250 \%$ (in relation to Spray and Wait).

PROPHET takes into account the frequency of past interactions to decide on replication. However, having a high number of past interactions does not mean nodes will have enough time and suitable conditions to exchange information when they meet again. For instance, a stationary node may be in the the path of a bus which results in a high frequency of past contacts; still, speed and physical obstacles such as office walls may lead to a problematic link seen by the proposal as a good exchange opportunity (i.e., high delivery predictability). Thus, unwanted copies (up to $\sim 1260$ per delivery) are generated to reach just a little over 55\% delivery probability and that take up to $96 \%$ (compared to Spray and Wait) more time to reach destinations.

Epidemic, considered as an upper bound to delivery probability, shows a different behavior: delivery probability up to $\sim 38 \%$ with the highest cost per delivered message (up to $\sim 2225$ copies) but with lower latency (up to $\sim 22 \%$ compared to Spray and Wait). This is due to the fact that the proposal does not worry about resource constraints, and this scenario is also challenging given the small amount of buffer space nodes are willing to share (i.e., 2MB).

As for the second scenario, trace-based, besides the buffer limitations, it is even more challenging since contacts are sporadic and much less (32) when compared to the heterogeneous scenario (962). This consequently increases the time proposals need to have a more suitable view of the network (in terms of the forwarding metrics they consider), thus increasing the time to decide on replication and to deliver messages.

The advantage of Spray and Wait reduced in this scenario where it achieves up to $35 \%$ of delivery with an increased cost of 15.37 copies per message delivered and latency increases going up to 44.372 seconds.

All the social-aware proposals keep a very close behavior to one another: reaching up to 33\% (dLifeComm) and 34\% (dLife and Bubble Rap) delivery predictability with a cost per delivery going up to $\sim 31$ and 25 copies and latency increases up to $\sim 20 \%$ (dLifeComm) and $\sim 24 \%$ (dLife and Bubble Rap) when compared to Spray and Wait.

Epidemic and PROPHET suffer even more with the sporadicity of contacts in this scenario, reaching up to $19 \%$ and $32 \%$ for delivery probabilities with an associated 
cost up to 987 and 536 copies per successful delivery and latency increases up to $\sim 16 \%$ and $\sim 31 \%$ when compared to Spray and Wait, respectively.

With these results one can conclude that still there is much work to be done in the sense of creating a new opportunistic routing solution be it based on social- oblivious or social-aware approaches. What is more, social-aware solutions show a great potential in improving forwarding in opportunistic networks. It is clear that there is a tradeoff between delivery probability/cost and latency, since these proposals take more time to choose the best next hop. It is important to note that the performance of social-oblivious solutions such as Spray and Wait still require further investigation as it has strong requirements. For instance, the number of spraying copies $L$, during our experiments was set statically. However, according to its paper [35], this parameter depends on the number of nodes in the network and must be estimated, and the current implementation used in our experiments does not take into account the effect of this.

Additionally, none of the proposals consider (multi)point-to-multipoint communication, which is a feature that should be looked upon given the need to efficiently spread content in opportunistic networks.

As mentioned before, our goal is not to elect the best solution, but instead to show the pros and cons of each of them, and most importantly to show that socialaware solutions deserve attention when it comes to developing routing approaches for opportunistic networks.

\section{Conclusions}

Social similarity has gained attention in the last years in the context of opportunistic networks given its potential to improve data forwarding. The reason behind this is that devices are carried by humans who happen to have different aspects who may help to identify one or many of them. Such aspects can be related to social ties, work affiliation, shared interests, and among others, that can be used to infer/find a social similarity. In addition, routing based on social similarity has proven to be much more stable that those based on mobility update (it is less volatile).

Thus, this chapter aims at introducing the new trend observed since 2007 with the appearance of social-aware opportunistic routing. For that, we cover a 12-year period worth of opportunistic routing solutions starting with a close look in the social-oblivious one to help us in understanding the need for this new trend. Then, we follow to a close look at pioneer social-aware solutions along with new ones, covering how they use social similarity to devise their forwarding approaches.

The chapter also provides a brief look at the existing opportunistic routing taxonomies, in order to show when the social trend appeared and how much importance is given to it. We also take this opportunity to propose an update to an existing taxonomy, which includes the appearance of a new sub-category of social similarity solutions based on user dynamic behavior. 
Finally, the chapter includes a set of experiments in different scenarios pointing out advantages and disadvantages of each social-oblivious and social-aware proposal, and indeed showing the potential of social-aware solutions to support our case: show that the trend is strong and deserves careful attention from researchers.

\section{References}

1. Balasubramanian, A., Levine, B., Venkataramani, A.: Dtn routing as a resource allocation problem. In: Proceedings of the 2007 conference on Applications, technologies, architectures, and protocols for computer communications, SIGCOMM '07, pp. 373-384. ACM, New York, NY, USA (2007). DOI 10.1145/1282380.1282422. URL http://doi.acm.org/10.1145/1282380.1282422

2. Basagni, S., Chlamtac, I., Syrotiuk, V.R., Woodward, B.A.: A distance routing effect algorithm for mobility (dream). In: Proceedings of the 4th annual ACM/IEEE international conference on Mobile computing and networking, MobiCom '98, pp. 7684. ACM, New York, NY, USA (1998). DOI 10.1145/288235.288254. URL http://doi.acm.org/10.1145/288235.288254

3. Bloom, B.H.: Space/time trade-offs in hash coding with allowable errors. Commun. ACM 13(7), 422-426 (1970). DOI 10.1145/362686.362692. URL http://doi.acm.org/10.1145/362686.362692

4. Burgess, J., Gallagher, B., Jensen, D., Levine, B.N.: Maxprop: Routing for vehicle-based disruption-tolerant networks. In: INFOCOM 2006. 25th IEEE International Conference on Computer Communications. Proceedings, pp. 1 -11 (2006). DOI 10.1109/INFOCOM.2006. 228

5. Cerf, V., Burleigh, S., Hooke, A., Torgerson, L., Durst, R., Scott, K., Fall, K., Weiss, H.: Delay tolerant network architecture. IETF Network Working Group. RFC 4838 (2007)

6. Chaintreau, A., Hui, P., Crowcroft, J., Diot, C., Gass, R., Scott, J.: Impact of human mobility on opportunistic forwarding algorithms. IEEE Transactions on Mobile Computing 6(6), 606-620 (2007). DOI 10.1109/TMC.2007.1060. URL http://dx.doi.org/10.1109/TMC.2007.1060

7. Costa, P., Mascolo, C., Musolesi, M., Picco, G.P.: Socially-aware routing for publish-subscribe in delay-tolerant mobile ad hoc networks. IEEE J.Sel. A. Commun. 26(5), 748-760 (2008). DOI 10.1109/JSAC.2008.080602. URL http://dx.doi.org/10.1109/JSAC.2008.080602

8. Daly, E.M., Haahr, M.: Social network analysis for routing in disconnected delay-tolerant manets. In: Proceedings of the 8th ACM international symposium on Mobile ad hoc networking and computing, MobiHoc '07, pp. 32-40. ACM, New York, NY, USA (2007). DOI 10.1145/1288107.1288113. URL http://doi.acm.org/10.1145/1288107.1288113

9. D'Souza, R.J., Jose, J.: Routing approaches in delay tolerant networks: A survey. International Journal of Computer Applications 1(17), 8-14 (2010). Foundation of Computer Science

10. Dubois-Ferriere, H., Grossglauser, M., Vetterli, M.: Age matters: efficient route discovery in mobile ad hoc networks using encounter ages. In: Proceedings of the 4th ACM international symposium on Mobile ad hoc networking \& computing, MobiHoc '03, pp. 257-266. ACM, New York, NY, USA (2003). DOI 10.1145/778415.778446. URL http://doi.acm.org/10.1145/778415.778446

11. Grasic, S., Davies, E., Lindgren, A., Doria, A.: The evolution of a dtn routing protocol prophetv2. In: Proceedings of the 6th ACM workshop on Challenged networks, CHANTS '11, pp. 27-30. ACM, New York, NY, USA (2011). DOI 10.1145/2030652.2030661. URL http://doi.acm.org/10.1145/2030652.2030661 
12. Grossglauser, M., Vetterli, M.: Locating mobile nodes with ease: learning efficient routes from encounter histories alone. IEEE/ACM Trans. Netw. 14(3), 457-469 (2006). DOI 10.1109/ TNET.2006.876204. URL http://dx.doi.org/10.1109/TNET.2006.876204

13. Hossmann, T., Spyropoulos, T., Legendre, F.: Know thy neighbor: towards optimal mapping of contacts to social graphs for dtn routing. In: Proceedings of the 29th conference on Information communications, INFOCOM'10, pp. 866-874. IEEE Press, Piscataway, NJ, USA (2010). URL http://dl.acm.org/citation.cfm?id=1833515.1833658

14. Hui, P.: People are the network: experimental design and evaluation of social-based forwarding algorithms. Tech. Rep. UCAM-CL-TR-713, University of Cambridge, Computer Laboratory (2008)

15. Hui, P., Crowcroft, J.: How small labels create big improvements. In: Pervasive Computing and Communications Workshops, 2007. PerCom Workshops '07. Fifth Annual IEEE International Conference on, pp. 65 -70 (2007). DOI 10.1109/PERCOMW.2007.55

16. Hui, P., Crowcroft, J., Yoneki, E.: Bubble rap: social-based forwarding in delay tolerant networks. In: Proceedings of the 9th ACM international symposium on Mobile ad hoc networking and computing, MobiHoc '08, pp. 241-250. ACM, New York, NY, USA (2008). DOI 10.1145/ 1374618.1374652. URL http://doi.acm.org/10.1145/1374618.1374652

17. Hui, P., Crowcroft, J., Yoneki, E.: Bubble rap: Social-based forwarding in delay-tolerant networks. IEEE Transactions on Mobile Computing 10(11), 1576-1589 (2011). DOI 10.1109/TMC.2010.246. URL/http://dx.doi.org/10.1109/TMC.2010.246

18. Jaho, E., Karaliopoulos, M., Stavrakakis, I.: Social similarity as a driver for selfish, cooperative and altruistic behavior. In: Proceedings of the 2010 IEEE International Symposium on A World of Wireless, Mobile and Multimedia Networks (WoWMoM), WOWMOM '10, pp. 16. IEEE Computer Society, Washington, DC, USA (2010). DOI 10.1109/WOWMOM.2010. 5534930. URL/http://dx.doi.org/10.1109/WOWMOM.2010.5534930

19. Jain, S., Fall, K., Patra, R.: Routing in a delay tolerant network. In: Proceedings of the 2004 conference on Applications, technologies, architectures, and protocols for computer communications, SIGCOMM '04, pp. 145-158. ACM, New York, NY, USA (2004). DOI 10.1145/ 1015467.1015484. URL http://doi.acm.org/10.1145/1015467.1015484

20. Jones, E.P.C., Li, L., Ward, P.A.S.: Practical routing in delay-tolerant networks. In: Proceedings of the 2005 ACM SIGCOMM workshop on Delay-tolerant networking, WDTN '05, pp. 237-243. ACM, New York, NY, USA (2005). DOI 10.1145/1080139.1080141. URL http://doi.acm.org/10.1145/1080139.1080141

21. Karamshuk, D., Boldrini, C., Conti, M., Passarella, A.: An arrival-based framework for human mobility modeling. In: World of Wireless, Mobile and Multimedia Networks (WoWMoM), 2012 IEEE International Symposium on a, pp. 1 -9 (2012). DOI 10.1109/WoWMoM.2012. 6263683

22. Keränen, A., Ott, J., Kärkkäinen, T.: The one simulator for dtn protocol evaluation. In: Proceedings of the 2nd International Conference on Simulation Tools and Techniques, Simutools '09, pp. 55:1-55:10. ICST (Institute for Computer Sciences, Social-Informatics and Telecommunications Engineering), ICST, Brussels, Belgium, Belgium (2009). DOI 10.4108/ICST.SIMUTOOLS2009.5674. URL http://dx.doi.org/10.4108/ICST.SIMUTOOLS2009.5674

23. Lindgren, A., Doria, A., Schelén, O.: Probabilistic routing in intermittently connected networks. SIGMOBILE Mob. Comput. Commun. Rev. 7(3), 19-20 (2003). DOI 10.1145/961268. 961272. URL http://doi.acm.org/10.1145/961268.961272

24. Liu, C., Wu, J.: An optimal probabilistic forwarding protocol in delay tolerant networks. In: Proceedings of the tenth ACM international symposium on Mobile ad hoc networking and computing, MobiHoc '09, pp. 105-114. ACM, New York, NY, USA (2009). DOI 10.1145/ 1530748.1530763. URL http://doi.acm.org/10.1145/1530748.1530763

25. Moreira, W., Mendes, P.: Survey on opportunistic routing for delay/disruption tolerant networks. Tech. Rep. SITI-TR-11-02, Research Unit in Informatics Systems and Technologies (SITI), University Lusofona (2011) 
26. Moreira, W., Mendes, P., Sargento, S.: Assessment model for opportunistic routing. In: Communications (LATINCOM), 2011 IEEE Latin-American Conference on, pp. 1 -6 (2011). DOI 10.1109/LatinCOM.2011.6107393

27. Moreira, W., Mendes, P., Sargento, S.: Assessment model for opportunistic routing. Latin America Transactions, IEEE (Revista IEEE America Latina) 10(3), 1785 -1790 (2012). DOI 10.1109/TLA.2012.6222585

28. Moreira, W., Mendes, P., Sargento, S.: Opportunistic routing based on daily routines. In: World of Wireless, Mobile and Multimedia Networks (WoWMoM), 2012 IEEE International Symposium on a, pp. 1 -6 (2012). DOI 10.1109/WoWMoM.2012.6263749

29. Mtibaa, A., May, M., Diot, C., Ammar, M.: Peoplerank: social opportunistic forwarding. In: Proceedings of the 29th conference on Information communications, INFOCOM'10, pp. 111-115. IEEE Press, Piscataway, NJ, USA (2010). URL http://dl.acm.org/citation.cfm?id=1833515.1833538

30. Nelson, S., Bakht, M., Kravets, R.: Encounter-based routing in dtns. In: INFOCOM 2009, IEEE, pp. 846 -854 (2009). DOI 10.1109/INFCOM.2009.5061994

31. Nguyen, H.A., Giordano, S.: Context information prediction for social-based routing in opportunistic networks. Ad Hoc Netw. 10(8), 1557-1569 (2012). DOI 10.1016/j.adhoc.2011. 05.007. URL http://dx.doi.org/10.1016/j.adhoc.2011.05.007

32. Ramanathan, R., Hansen, R., Basu, P., Rosales-Hain, R., Krishnan, R.: Prioritized epidemic routing for opportunistic networks. In: Proceedings of the 1st international MobiSys workshop on Mobile opportunistic networking, MobiOpp '07, pp. 6266. ACM, New York, NY, USA (2007). DOI 10.1145/1247694.1247707. URL http://doi.acm.org/10.1145/1247694.1247707

33. Scott, J., Gass, R., Crowcroft, J., Hui, P., Diot, C., Chaintreau, A.: CRAWDAD trace cambridge/haggle/imote/content (v. 2006-09-15). Downloaded from http://crawdad.cs.dartmouth.edu/cambridge/haggle/imote/content (2006)

34. Song, L., Kotz, D.F.: Evaluating opportunistic routing protocols with large realistic contact traces. In: Proceedings of the second ACM workshop on Challenged networks, CHANTS '07, pp. 35-42. ACM, New York, NY, USA (2007). DOI 10.1145/1287791.1287799. URL http://doi.acm.org/10.1145/1287791.1287799

35. Spyropoulos, T., Psounis, K., Raghavendra, C.S.: Spray and wait: an efficient routing scheme for intermittently connected mobile networks. In: Proceedings of the 2005 ACM SIGCOMM workshop on Delay-tolerant networking, WDTN '05, pp. 252259. ACM, New York, NY, USA (2005). DOI 10.1145/1080139.1080143. URL http://doi.acm.org/10.1145/1080139.1080143

36. Spyropoulos, T., Psounis, K., Raghavendra, C.S.: Spray and focus: Efficient mobility-assisted routing for heterogeneous and correlated mobility. In: Proceedings of the Fifth IEEE International Conference on Pervasive Computing and Communications Workshops, PERCOMW '07, pp. 79-85. IEEE Computer Society, Washington, DC, USA (2007). DOI 10.1109/ PERCOMW.2007.108. URL/http://dx.doi.org/10.1109/PERCOMW.2007.108

37. Spyropoulos, T., Psounis, K., Raghavendra, C.S.: Efficient routing in intermittently connected mobile networks: the single-copy case. IEEE/ACM Trans. Netw. 16(1), 63-76 (2008). DOI 10.1109/TNET.2007.897962. URL http://dx.doi.org/10.1109/TNET.2007.897962

38. Spyropoulos, T., Rais, R.N., Turletti, T., Obraczka, K., Vasilakos, A.: Routing for disruption tolerant networks: taxonomy and design. Wirel. Netw. 16(8), 2349-2370 (2010). DOI 10.1007/s11276-010-0276-9. URL http://dx.doi.org/10.1007/s11276-010-0276-9

39. Vahdat, A., Becker, D.: Epidemic routing for partially connected ad hoc networks. Tech. Rep. CS-200006, Duke University (2000)

40. Zhang, Z.: Routing in intermittently connected mobile ad hoc networks and delay tolerant networks: overview and challenges. Commun. Surveys Tuts. 8(1), 24-37 (2006). DOI 10.1109/COMST.2006.323440. URL http://dx.doi.org/10.1109/COMST.2006.323440 
41. Zhao, W., Ammar, M., Zegura, E.: Multicasting in delay tolerant networks: semantic models and routing algorithms. In: Proceedings of the 2005 ACM SIGCOMM workshop on Delay-tolerant networking, WDTN '05, pp. 268-275. ACM, New York, NY, USA (2005). DOI 10.1145/1080139.1080145. URL http://doi.acm.org/10.1145/1080139.1080145 